\title{
ESPRESSO at VLT
}

\section{On-sky performance and first results ${ }^{\star}$}

\author{
F. Pepe ${ }^{1}$, S. Cristiani ${ }^{2,16}$, R. Rebolo ${ }^{3,18,19}$, N. C. Santos ${ }^{4,12}$, H. Dekker ${ }^{5}$, A. Cabral ${ }^{6,11}$, P. Di Marcantonio ${ }^{2}$, \\ P. Figueira ${ }^{4,15}$, G. Lo Curto ${ }^{15}$, C. Lovis $^{1}$, M. Mayor ${ }^{1}$, D. Mégevand ${ }^{1}$, P. Molaro $^{2,16}$, M. Riva $^{7}$,
} M. R. Zapatero Osorio ${ }^{8}$, M. Amate ${ }^{3}$, A. Manescau ${ }^{5}$, L. Pasquini ${ }^{5}$, F. M. Zerbi ${ }^{7}$, V. Adibekyan ${ }^{4,12}$, M. Abreu ${ }^{6,11}$, M. Affolter ${ }^{9}$, Y. Alibert ${ }^{9}$, M. Aliverti ${ }^{7}$, R. Allart ${ }^{1}$, C. Allende Prieto ${ }^{3,18}$, D. Álvarez ${ }^{5}$, D. Alves ${ }^{6,11}$, G. Avila ${ }^{5}$, V. Baldini ${ }^{2}$, T. Bandy ${ }^{9}$, S. C. C. Barros ${ }^{4}$, W. Benz ${ }^{9}$, A. Bianco ${ }^{7}$, F. Borsa ${ }^{7}$, V. Bourrier ${ }^{1}$, F. Bouchy ${ }^{1}$, C. Broeg ${ }^{9}$, G. Calderone ${ }^{2}$, R. Cirami ${ }^{2}$, J. Coelho ${ }^{6,11}$, P. Conconi ${ }^{7}$, I. Coretti ${ }^{2}$, C. Cumani ${ }^{5}$, G. Cupani ${ }^{2}$, V. D'Odorico ${ }^{2,16}$, M. Damasso ${ }^{10}$, S. Deiries ${ }^{5}$, B. Delabre ${ }^{5}$, O. D. S. Demangeon ${ }^{4}$, X. Dumusque ${ }^{1}$, D. Ehrenreich ${ }^{1}$, J. P. Faria ${ }^{4,12}$, A. Fragoso ${ }^{3}$, L. Genolet ${ }^{1}$, M. Genoni ${ }^{7}$, R. Génova Santos ${ }^{3,18}$, J. I. González Hernández ${ }^{3,18}$, I. Hughes ${ }^{1}$, O. Iwert ${ }^{5}$, F. Kerber ${ }^{5}$, J. Knudstrup ${ }^{5}$, M. Landoni ${ }^{7}$, B. Lavie ${ }^{1}$, J. Lillo-Box ${ }^{8}$, J.-L. Lizon ${ }^{5}$, C. Maire ${ }^{1}$, C. J. A. P. Martins ${ }^{4,17}$, A. Mehner ${ }^{15}$, G. Micela ${ }^{13}$, A. Modigliani ${ }^{5}$, M. A. Monteiro ${ }^{4}$, M. J. P. F. G. Monteiro ${ }^{4,12}$, M. Moschetti ${ }^{7}$, M. T. Murphy ${ }^{20}$, N. Nunes ${ }^{6,11}$, L. Oggioni ${ }^{7}$, A. Oliveira ${ }^{6,11}$, M. Oshagh ${ }^{3}$, E. Pallé ${ }^{3,18}$, G. Pariani ${ }^{7}$, E. Poretti ${ }^{7}$, J. L. Rasilla ${ }^{3}$, J. Rebordão ${ }^{6,11}$, E. M. Redaelli ${ }^{7}$, S. Santana Tschudi ${ }^{3,5}$, P. Santin ${ }^{2}$, P. Santos ${ }^{6,11}$, D. Ségransan ${ }^{1}$, T. M. Schmidt ${ }^{2}$, A. Segovia ${ }^{1}$, D. Sosnowska ${ }^{1}$, A. Sozzetti ${ }^{10}$, S. G. Sousa ${ }^{4}$, P. Spanò ${ }^{14}$, A. Suárez Mascareño ${ }^{3,18}$, H. Tabernero ${ }^{4}$, F. Tenegi ${ }^{3}$, S. Udry ${ }^{1}$, and A. Zanutta ${ }^{7}$

(Affiliations can be found after the references)

Received 30 April 2020 / Accepted 29 September 2020

\section{ABSTRACT}

Context. ESPRESSO is the new high-resolution spectrograph of ESO's Very Large Telescope (VLT). It was designed for ultra-high radial-velocity (RV) precision and extreme spectral fidelity with the aim of performing exoplanet research and fundamental astrophysical experiments with unprecedented precision and accuracy. It is able to observe with any of the four Unit Telescopes (UTs) of the VLT at a spectral resolving power of 140000 or 190000 over the 378.2 to $788.7 \mathrm{~nm}$ wavelength range; it can also observe with all four UTs together, turning the VLT into a $16 \mathrm{~m}$ diameter equivalent telescope in terms of collecting area while still providing a resolving power of 70000 .

Aims. We provide a general description of the ESPRESSO instrument, report on its on-sky performance, and present our Guaranteed Time Observation (GTO) program along with its first results.

Methods. ESPRESSO was installed on the Paranal Observatory in fall 2017. Commissioning (on-sky testing) was conducted between December 2017 and September 2018. The instrument saw its official start of operations on October 1, 2018, but improvements to the instrument and recommissioning runs were conducted until July 2019.

Results. The measured overall optical throughput of ESPRESSO at $550 \mathrm{~nm}$ and a seeing of $0.65^{\prime \prime}$ exceeds the $10 \%$ mark under nominal astroclimatic conditions. We demonstrate an RV precision of better than $25 \mathrm{~cm} \mathrm{~s}^{-1}$ during a single night and $50 \mathrm{~cm} \mathrm{~s}^{-1}$ over several months. These values being limited by photon noise and stellar jitter shows that the performance is compatible with an instrumental precision of $10 \mathrm{~cm} \mathrm{~s}^{-1}$. No difference has been measured across the UTs, neither in throughput nor RV precision.

Conclusions. The combination of the large collecting telescope area with the efficiency and the exquisite spectral fidelity of ESPRESSO opens a new parameter space in RV measurements, the study of planetary atmospheres, fundamental constants, stellar characterization, and many other fields.

Key words. techniques: radial velocities - instrumentation: spectrographs - planets and satellites: atmospheres - planets and satellites: detection cosmology: miscellaneous - asteroseismology

\section{Introduction}

High-resolution spectroscopy enables key physical insight into the study of stars, galaxies, and interstellar and intergalactic media. High precision and high dispersion have emerged in recent years as crucial elements in the spectroscopy of faint objects, which called in turn for the use of larger telescopes to increase the photon-collecting area. Furthermore, many science

\footnotetext{
^ Based on GTOs collected at the European Southern Observatory under ESO program(s) 1102.C-0744, 1102.C-0958 and 1104.C-0350 by the ESPRESSO Consortium.
}

cases require repeatable observations over a long temporal baseline. For instance, the HARPS spectrograph at the ESO $3.6 \mathrm{~m}$ telescope has established itself as a pioneering instrument for radial-velocity (RV) measurements (Mayor et al. 2003) because of its ultra-high precision, as well as its operational efficiency and continuous availability on a medium-size telescope.

The search for and characterization of terrestrial planets in the habitable zone (HZ) of their parent stars will be one of the most exciting science topics of the coming decades and one of the main science drivers for the new generation of space missions and extremely large telescopes. These goals rely on the 
definition of a suitable target list that has been cleaned of false positives and is filled with good targets for follow-up and characterization. There is a gap between past observations and surveys with small- and medium-size telescopes ( $<4 \mathrm{~m}$ diameter) and the target-focused observations on future large facilities (e.g., the Extremely Large Telescope; ELT), which can and must be filled by the optimum use of new instruments on currently accessible 10-m class telescopes.

Another aspect that must be considered is the fast evolution of the field from exoplanet search to exoplanet characterization. A sudden change was induced by space-based transit searches such as CoRoT and Kepler. The latter in particular started delivering thousands of transiting candidates that had to be confirmed, and, more importantly, for which we were lacking mass measurements. This situation emphasized the synergy between RVs and transit observations, and thus the need for ground-based RV follow-up in view of better understanding the internal structure and composition of exoplanets. Unfortunately, most of the transiting candidates delivered by Kepler are too faint for RV followup with small and medium-size telescopes, and they are "even fainter" when attempts are made at observing planetary atmospheres by the means of ground-based transit spectroscopy.

These facts were recognized by the ESO-ESA working group report on extrasolar planets in 2005, which emphasized the need for HARPS-like instruments on large telescopes (Perryman et al. 2005). Simultaneously, the idea of a HARPS-like instrument for the Very Large Telescope (VLT) was directly derived from the CODEX project (Pasquini et al. 2005; Molaro et al. 2006; Molaro 2009), a phase-A study for the development of an ultrahigh-precision spectrograph for the European ELT that was presented by Pasquini et al. (2009) in 2007 at the conference Science with the VLT in the ELT Era. Following this idea, ESO's Scientific Technical Committee (STC) recommended the development of second-generation VLT instruments in October 2007, and this proposal was endorsed by the ESO Council in December of the same year. Among others, a high-resolution, ultra-stable spectrograph for the VLT combined-Coudé focus was suggested. Following these recommendations, ESO issued a call in March 2008 for proposals to carry out the Phase-A study for such an instrument driven by three main science cases: "(1) detection and characterization of rocky planets in the HZ; (2) study of the variability of fundamental constants; and (3) analysis of the chemical composition of stars in nearby galaxies". The proposal submitted by the ESPRESSO Consortium ${ }^{1}$ was accepted by ESO and the consortium was selected to carry out the construction of this spectrograph in collaboration with ESO as a partner. In exchange for funding and building the instrument, the ESPRESSO Consortium was awarded 273 nights of Guaranteed Time Observation (GTO) in order to pursue part of the science cases described above.

The present paper aims at providing a general description of the ESPRESSO instrument and its performance as delivered to the community by summer 2019 . Section 2 summarizes the project history. In Sect. 3, we present a first-level description of the instrument and its subsystems to provide a general overview without entering into technical details; instead, references to

\footnotetext{
The ESPRESSO Consortium was composed of: Observatoire Astronomique de l'Université de Genève (project head, Switzerland); Centro de Astrofísica da Universidade do Porto (Portugal); Faculdade de Ciencias da Universidade de Lisboa (Portugal); INAF-Osservatorio Astronomico di Brera (Italy); INAF-Osservatorio Astronomico di Trieste (Italy); Instituto de Astrofísica de Canarias (Spain); and Physikalisches Institut der Universität Bern (Switzerland). ESO participated in the ESPRESSO project as an associated partner.
}

technical papers will be given. Additionally, we will describe the current operation scheme of ESPRESSO at the Paranal Observatory. Section 4 focuses on the on-sky performance derived from the commissioning phases and the first periods of operation. In Sect. 5, we describe our GTO program and give an overview of suitable ESPRESSO science cases. Examples of the first results obtained during the initial periods of observation are presented, and references to the corresponding papers are given.

\section{The ESPRESSO project}

The project kick-off was held in February 2011, and the design phase ended with the final design review in May 2013. The procurement of components and manufacturing of subsystems took roughly three years, considerably longer than originally planned. Integration started in early 2016 with the first subsystem, the large vacuum vessel, in the integration hall of the Geneva Observatory, which overlapped with the procurement phase of other subsystems in the various consortium partner institutes. Assembly, integration, and verification (AIV) was completed with the Provisional Acceptance Europe (PAE) in June 2017. In parallel to the procurement and AIV phase of the spectrograph in Europe, the unexploited Combined-Coudé Laboratory (CCL) of the VLT was cleared and equipped with an air-conditioned environment to accept the ESPRESSO spectrograph. A considerable effort was devoted by the Consortium and ESO to equip all the Unit Telescopes (UTs) of the VLT with the so-called CoudéTrain (CT), an ensemble of optics, mechanics, and control units to route the light from each UT through the "incoherent" Coudépath and the tunnels to the CCL, where it can be either combined to use ESPRESSO in the 4-UT configuration or individually selected to feed the spectrograph in its one-UT configuration. Both the CCL (2015-2016) and the CT (October 2016) had been commissioned by the PAE and were ready to welcome the spectrograph, even though some UT branches of the CT would have to be commissioned and realigned later on during the commissioning of the spectrograph. The spectrograph was shipped from Geneva to Paranal in summer 2017.

During fall 2017, the on-site reassembly in the CCL started, followed by the integration with the CT. The CT had been commissioned to verify all interfaces, functionalities, and performance, especially with respect to telescope selection, optical path configuration, telescope presetting, pointing, acquisition, tracking, and guiding. ESPRESSO required on-sky configuration and testing on each of the UTs individually in the 1-UT configuration and on all four UTs together in the 4-UT configuration. The commissioning plan and strategy was adapted to scheduling requirements, and the commissioning runs were performed on the dates reported in Table 1.

First light of ESPRESSO in the 1-UT configuration took place on November 27, 2017, the date on which the official ESPRESSO commissioning started (ESO Release eso1739). This commissioning run was followed by others in January, February, April, and July 2018. During these runs, all modes of ESPRESSO (including the 4-UT configuration) were tested "on sky" and characterized. First light in the 4-UT configuration took place during the January 2018 run in the presence of ESO's director general (ESO Release eso1806). The simultaneous use of the four UTs implies a great complexity of the control system. Despite this, all processes - communication with the telescopes, configuration of the instrument in this new mode, slewing and pointing of the telescopes, acquisition of the target 
Table 1. Commissioning runs and technical missions on ESPRESSO.

\begin{tabular}{llll}
\hline \hline Commissioning run & Start date & End date & Tested modes and telescopes \\
\hline First light & $27 / 11 / 2017$ & & \\
Commissioning 1A & $27 / 11 / 2017$ & $06 / 12 / 2017$ & HR and UHR modes, all UTs \\
Commissioning 1B & $26 / 01 / 2018$ & $07 / 02 / 2018$ & All modes and all configurations \\
Commissioning 2A & $25 / 02 / 2018$ & $07 / 03 / 2018$ & All modes, all UTs \\
Technical activities & $07 / 03 / 2018$ & $21 / 03 / 2018$ & All subsystems \\
Commissioning 2B & $27 / 04 / 2018$ & $05 / 05 / 2018$ & HR and UHR mode, all UTs \\
Technical activities & $13 / 09 / 2018$ & $21 / 09 / 2018$ & Vacuum and cryogenics optimization \\
Official start of operations & $01 / 10 / 2018$ & & \\
Technical activities & $03 / 10 / 2018$ & $23 / 03 / 2018$ & Optical efficiency tests and re-alignment \\
Commissioning 2C - July 2018 & $03 / 07 / 2018$ & $09 / 07 / 2018$ & All modes and all configurations \\
4-UT Commissioning - December 2018 & $30 / 11 / 2018$ & $01 / 12 / 2018$ & MR mode, all UTs \\
Technical activities & $08 / 06 / 2019$ & $27 / 06 / 2019$ & Fiber upgrade \\
Recommissioning - July 2019 & $27 / 06 / 2019$ & $08 / 07 / 2019$ & All modes and all configurations \\
\hline
\end{tabular}

on all the telescopes, guiding, and exposing - were carried out without failure and produced, on the first attempt, the first visible high-resolution spectrum of an astronomical target obtained with the collecting area of a $16 \mathrm{~m}$ diameter telescope equivalent.

Minor improvements on hardware and software were performed in parallel to the commissioning phase, but the instrument demonstrated excellent operational performance and reliability from the beginning. While RV and stability requirements were essentially met, optical throughput suffered an unexpected deficiency. For this reason, the spectrograph was reopened and individual elements were optically tested in September 2018. It was found that the main culprit was the fiber link, but it was neither possible nor suitable to perform corrective actions on the spot because throughput and RV precision in the 1-UT configuration were satisfactory and the instrument was about to start operations. Two additional nights of 4-UT commissioning period took place in December 2018 in order to perform general functional and preliminary performance tests.

The official start of operations in 1-UT configuration took place on October 1, 2018, the date on which ESPRESSO was offered by ESO both to the community for open-time observations and to the ESPRESSO Consortium for GTO. During the first semester of operations, and in view of the start of operations in the 4-UT configuration by April 2019, the spare fiber link was sent back to Europe, repaired, and upgraded. Laboratory tests at the Osservatorio Astronomico di Brera in Merate immediately showed that an improvement in throughput was within reach. After returning the spare fiber link to Paranal, the component was installed and tested on the instrument in June 2019. This major intervention required a partial recommissioning of the 1-UT configuration and completion of the 4-UT configuration commissioning. Both were conducted successfully, despite bad weather conditions, from July 3 to 8, 2019. By July 9, 2019, the instrument could be considered to be in its final configuration and fully operational. No changes were made after this date apart from developing and implementing a new instrument mode - the HR42 mode, which is a standard-resolution mode with heavier binning and slow readout speed - with the aim of pushing the readout noise $(\mathrm{RON})$ limited magnitude range to fainter magnitudes. While the practical implementation of this mode in the instrument software turned out to be straightforward, the adaptation of the data reduction software demanded quite a bit of effort due to the different spectral format.

\section{The ESPRESSO instrument}

\subsection{Technical description of the instrument}

ESPRESSO is a fiber-fed, cross-dispersed, high-resolution echelle spectrograph located in the CCL at the incoherent focus of the VLT, where it can be fed by the light of either one UT (1-UT configuration) or all four UTs (4-UT configuration). In each of its configurations, two fibers illuminate the spectrograph: One fiber carries the light from the science target, and the second carries either the light from the sky background ( 7 " away) or the light from a reference source for simultaneous drift measurements. A detailed description of the instrument and all its subsystems is given in Pepe et al. (2014), Mégevand et al. (2014), González Hernández et al. (2018), and references therein. The control software (CS) and electronics are described more specifically in Calderone et al. (2016, 2018), Baldini et al. (2016). An overview of the scientific software and the description of its flow and products are presented in Sect. 3.3. The operational concept of ESPRESSO and its observing modes are outlined in Sect. 3.2.

\subsubsection{Light path from the telescope to the CCL}

The telescope light is brought to the CCL via the CT (Fig. 1). The CT is an all-optical system of prisms, mirrors, and lenses that guide the light from the Nasmyth focus through the elevation axis, the telescope structure, and the azimuth axis to the Coudé room (basement) of the specific telescope. There, the beam is bent and launched through the incoherent tunnels to the CCL, where the light passes through an atmospheric dispersion compensator (ADC). A detailed description of the highly complex CT can be found in Cabral et al. (2013, 2014, 2019). Its installation and alignment (Avila et al. 2016) represented a considerable share of the overall project effort, but it was necessary to transform ESPRESSO into the first (and only) instrument able to make use of the incoherent focus of the VLT, as such converting the VLT into the largest collecting-area optical telescope currently available.

\subsubsection{Front-end unit and calibration system}

In the CCL, the front end (FE) shapes and collects the light beams from one to four telescopes. Its design is described in Pepe et al. (2014) and Riva et al. (2014a). The FE is composed of four units, one per telescope, each of which serve 


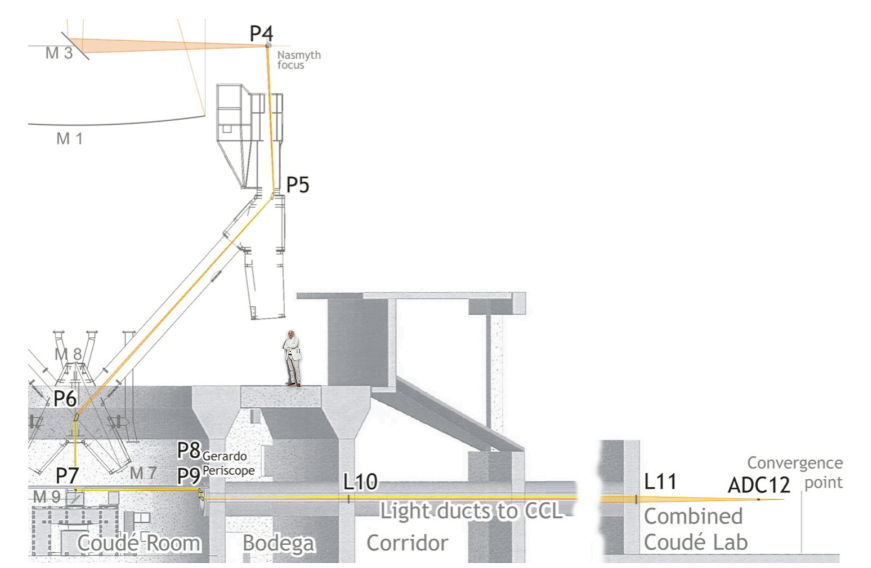

Fig. 1. Optical light path of the CT from the Nasmyth platform to the CCL. P4 to P7 are folding prisms that route the beam from the Nasmyth platform to the telescope's Coudé room. The mirrors R8 and R9 form a periscope that redirects the beam into the tunnel. At the entrance and the exit of the tunnel, respectively, the two lenses L10 and L11 recondition the light beam so it can enter the FE unit inside the CCL with a specified F-number (fixed for all telescopes). Two counter-rotating double prisms, ADC12, eventually correct for atmospheric dispersion across the wide spectral range of the spectrograph. This figure is from Pepe et al. (2014) and ESPRESSO Project documentation.

several functions: (1) a telescope-pupil stabilization by means of a pupil camera (Calderone et al. 2016) and a tip-tilt mirror; (2) sa field stabilization by means of a second tip-tilt mirror and a guiding camera (Calderone et al. 2016); (3) a focusing mechanism to optimize light injection into the optical fiber; and (4) a calibration-light injection system that selects whether the spectrograph fibers "see" the sky or the calibration light and allows for the injection of the light of any calibration source into any of the two fibers. Each FE was integrated and aligned "blindly" (i.e., using only very precise mechanical references) in Merate, Italy, and installed in Paranal, adjusting the FE as a whole with respect to the incoming light beam (Pariani et al. 2016; Aliverti et al. 2016).

One important role of the FE is to perform field stabilization (or secondary guiding) that corrects for the tip-tilt motion of the star not corrected by the primary stabilization of the telescope or introduced along the optical path of the CT at frequencies $<10 \mathrm{~Hz}$. The images acquired by the field-stabilization camera are analyzed by a control loop to: determine the difference in position of the stellar image with regard to that of the fiber entrance; compute the error; and apply the correction to the tip-tilt mirror. This system both optimizes slit efficiency and avoids RV effects due to off-centered light injection (Pepe et al. 2002).

The light from various calibration sources can be injected through the FE in any of the fibers in order to perform orderdefinition and spectral flat-fielding (white light), wavelength calibration (absolute spectral reference), and drift measurement (stable spectral source) in simultaneous-reference mode (Mayor et al. 2003; Pepe et al. 2002). The white light consists of the laser-driven light source (LDLS) EQ-99X from Energetiq. Wavelength calibration is obtained by combining ThAr-lamp spectra with a white-light illuminated Fabry-Pérot. The former provides accuracy, and the latter delivers a high number of uniform spectral emission lines across the whole spectrum that ensure "local" wavelength precision (see Sect. 3.3.2 as well as Cersullo et al. 2019 and references therein). All these sources are coupled with optical fibers to two calibration units (Mégevand et al. 2014), one for each fiber channel of the spectrograph. The calibration units have the functionality of attenuating the light of the calibration source and redirecting it through another optical fiber to the corresponding FE unit.

A laser frequency comb (LFC) is also available and should have replaced both the ThAr lamp and the Fabry-Pérot (Pasquini \& Hubin 2018; Frank et al. 2018; Huke et al. 2018); however, due to a lack of reliability and limited spectral coverage, it is currently not integrated in the operational scheme. At the time of writing, it is still unclear when the LFC will become operational. It must be noted that, given the currently limited wavelength range, the LFC will not be used alone for complete wavelength calibration. This situation might compromise the ability of ESPRESSO to guarantee RV repeatability at the $10 \mathrm{~cm} \mathrm{~s}^{-1}$-level over years, since the wavelength calibration will have to rely on hollow-cathode lamps.

A toggling system (rotational plate) in the FE carries six fiber heads. Four fiber heads belong to the medium-resolution (MR) mode and can be positioned simultaneously at the output of the respective FE unit of each telescope in order to operate ESPRESSO in the 4-UT configuration. Alternatively, in the 1-UT configuration, either the high-resolution (HR) or the ultrahigh-resolution (UHR) head can be positioned by rotating the toggling system at the output of any of the four FE units.

Each fiber head carries a pierced mirror (Gracia Temich et al. 2018a) located in the focal plane of the FE unit. It reflects the light from the telescope to the field and pupil cameras. Figure 2 shows an image of the pierced focal mirror, the "field," as seen by the field-stabilization or secondary-guiding camera. The size of the field is about $17^{\prime \prime}$ in diameter, the distance between fiber A and fiber B is $7^{\prime \prime}$, and the scale-plate is $0.041^{\prime \prime}$ per $(2 \times 2$-binned $)$ pixel.

\subsubsection{Fiber feed}

At the back of each of the two pinholes in the mirror, relay optics re-image the light entering the pinholes onto two octagonal fibers, the target fiber and the reference fiber (for simplicity, called fiber A and B, respectively, hereafter). Each fiber individually carries the light to the double scrambler, which is mounted on the vacuum vessel. The main functionality of the double scrambler is to convert the far-field of the incoming optical fiber into the near field, and vice versa, while injecting the beam into a second section of an identical fiber. This system ensures, in combination with the use of octagonal fibers, a homogeneous and stable illumination of the spectrograph, which is fundamental for repeatable RV measurements (Chazelas et al. 2010, 2012; Cosentino et al. 2012). The double scrambler also integrates a vacuum window that provides the optical vacuum feedthrough of the scientific light. The exposure shutters, one for each mode, are installed on the air-side of the feedthrough within the double scrambler mechanics. In the 4-UT configuration, the double scrambler also has the task of combining the light from the four fibers of each channel, A and B, from the four telescopes into one single square-shaped fiber for each channel (Gracia Temich et al. 2018b). All the fibers from the scrambler are then routed to the focal plane of the spectrograph to form a single entrance "slit" (see the right-hand side of Fig. 4). The two fibers, A and B, of each mode are aligned vertically along the cross-dispersion direction, while all $\mathrm{A}$ and all B fibers are arranged side-by-side horizontally along the main-dispersion direction. The three separate exposure shutters for the three modes ensure that only one mode feeds the 


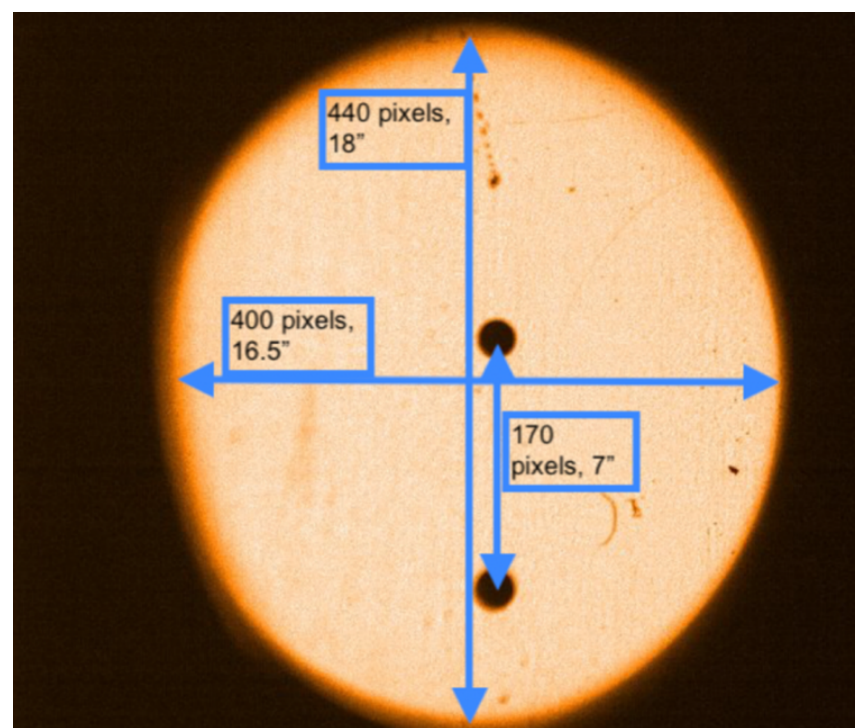

Fig. 2. Image of the pierced focal mirror, field, as seen by the fieldstabilization or secondary-guiding camera. The physical direction is rotated by $90^{\circ}$.

spectrograph at a time. The fiber-link design was based on the concepts originally developed for HARPS and HARPS-N (Cosentino et al. 2012; Lo Curto et al. 2015) and was adapted to ESPRESSO with its various observing and instrument modes (Mégevand et al. 2014).

\subsubsection{The spectrograph}

The light of the fibers entering the spectrograph is dispersed, and the spectra are imaged through two different camera arms, a red and a blue one, onto two distinct detectors. First, an anamorphic pupil slicing unit (APSU, Riva et al. 2014b; Oggioni et al. 2016) shapes the beam in order to reduce the size of the spectrograph optics; without the APSU, the size of the echelle grating would have to be $60 \times 240 \mathrm{~cm}$ to achieve the desired spectral resolution. Instead, the APSU slices and superimposes the produced half-pupils in the main dispersion direction, while in the other direction it anamorphically compresses the beam by a factor of 3 . The collimator, an off-axis parabola used in double pass, collimates the beam to a rectangular shape of $20 \times 30 \mathrm{~cm}$ that matches the $20 \times 120 \mathrm{~cm}$ physical surface of the $76^{\circ}(\mathrm{R} 4)$ echelle mosaic (Lizon et al. 2018). A dichroic beamsplitter placed before the intermediate spectrum sends the light to the blue and the red channels. For each channel, a specific (spherical) transfer collimator re-images the white pupil of the echelle grating onto the cross-dispersers, a volume-phase holographic (VPH) grating (Arns 2016). Two camera lenses eventually focus the blue and red portions of cross-dispersed echelle spectra separately, each onto its own large CCD detector.

Compared to a classical UVES-like white pupil design, ESPRESSO uses several design innovations: (1) By adjusting the power of the red field lens and the blue field mirror at the intermediate focal planes (white spectrum), the field curvature is compensated, thus avoiding the need for a cylindrical field lens in front of the detector. (2) The transfer collimator is a tilted sphere with a focal length much shorter than the main collimator; this significantly reduces the beam size on the cross-dispersers (asymmetric white pupil). (3) By adjusting the tilt angle of the spherical mirrors and deliberately operating

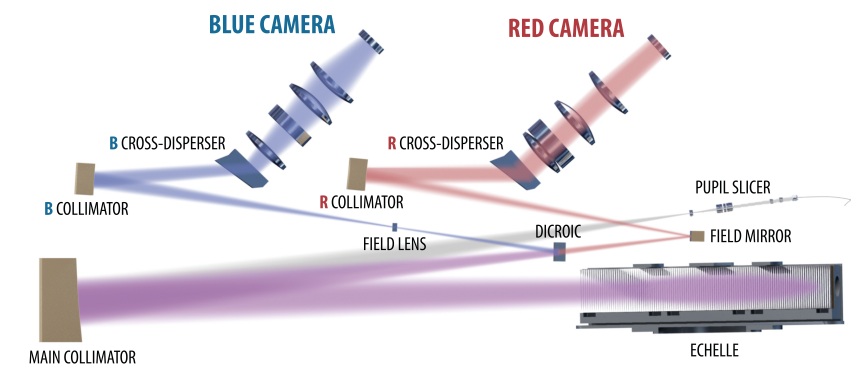

Fig. 3. Schematic view of the optical design and layout of the spectrograph and its components. This figure is from González Hernández et al. (2018), courtesy of Gonzalez-Hernandez.

the VPH cross-disperser in a non-collimated beam, aberrations introduced by the main collimator are compensated for, with the exception of the residual spherical aberration that is corrected for in the camera lenses. (4) A prism cemented to the VPH lifts the residual 1:1.5 anamorphism and returns a square beam. Furthermore, it compresses the spectral orders in the cross-dispersion direction by the same factor (1.5), reducing spatial sampling and needed CCD space. (5) The camera lenses each consist of only three lenses of inexpensive glass, the last of which is used as the cryostat window. In each camera, the residual longitudinal chromatism is compensated by titling the CCD chip.

Two individual E2V CCDs of $9 k \times 9 k$ and $10 \mu \mathrm{m}$-square pixels record the blue and red spectra separately. The CCDs have different thicknesses and were optimized in sensitivity for the blue and the red channels separately. The two chips are cooled and stabilized in temperature to $160 \pm 0.01 \mathrm{~K}$ by two individual continuous-flow cryostats (Lizon et al. 2016) that provide the detectors with separate high vacuum within the large ESPRESSO detector vessel. The CCDs are controlled and read out by ESO's New Generation Controllers (NGCs), which provide selectable readout speed, gain, and binning. Special attention was also paid to equalize the power consumption of the large CCD detectors during the different phases of idling, integrating, and reading through dummy clocking and dithering (Iwert et al., internal review documentation) so as to avoid "thermal breathing" of the pixel grid, as is seen in the HARPS detector mosaic. A schematic view of the spectrograph and its optical design is shown in Fig. 3.

\subsubsection{Spectral format}

Thanks to the use of a pupil slicer, the spectrograph delivers two simultaneous spectra of the light from the two fibers in a wavelength range between 378.2 and $788.7 \mathrm{~nm}$ and at a resolving power reaching $R>190000$ in the UHR mode and $R \sim 140000$ in the HR mode, the two instrument modes available in the 1-UT configuration. In the 4-UT configuration, also called MR mode, that combines the light collected by all the UTs, the same wavelength range is covered, but the increased etendue of the telescope comes at the price of a larger input fiber and thus a reduced resolving power of $R \sim 70000$. This value is nevertheless remarkable and exceptional, considering that ESPRESSO is the first optical spectrograph working with a $16 \mathrm{~m}$ diameter equivalent telescope.

Figure 4 shows the spectral formats of the blue and red arms. The traces represent the individual echelle orders over one free-spectral range (FSR). The right-hand panels show a zoomin on the "pseudo-slit" (combination of all fibers entering the spectrograph) re-imaged on the CCDs. A tabulated extract of 


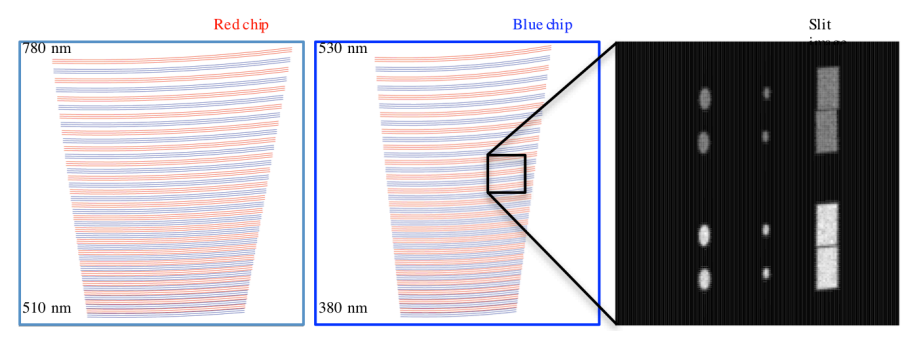

Fig. 4. Left: spectral format of the spectrum on the two detectors $(90 \times 90 \mathrm{~mm})$ showing traces of the various orders over one FSR. To better distinguish neighboring orders, even and odd order numbers have been drawn in blue and red, respectively. Each order is represented by three traces indicating the center of the order in the cross-dispersion direction (vertical), as well as the upper and lower boundaries of the full field produced by the pseudo-slit. Right: zoom-in of the virtual pseudoslit showing the images of the various fibers projected on the CCDs as if they were simultaneously illuminated by a monochromatic light source. The image covers a square of approximately 120 pixels $(1.2 \mathrm{~mm})$ in size on the detector. From left to right: images of the HR, UHR, and MR fibers. For each mode, the lower two images arise from the two slices of fiber A (target) and the upper two from fiber B (reference). In the actual application, only one mode is illuminated at a time, leading to four images (two fiber images $\times$ two slices) aligned along the vertical direction (cross-dispersion). The total height of the image determines the width of the echelle order and thus the required cross-dispersion needed to avoid the overlap of neighboring orders on the blue end of the spectral range. This figure is from Pepe et al. (2014).

wavelengths and spectral bin values is given in Table 2. The complete and detailed description of the spectral format can be obtained through the official Exposure Time Calculator (ETC) ${ }^{2}$.

\subsubsection{Exposure meter}

In order to track the flux entering the spectrograph in real time, and thus monitor the instantaneous efficiency, the part of the spectrograph beam that would fall outside the clear aperture of the spectrograph is collected within the APSU by a flat mirror with a central hole (pupil stop for the spectrograph). The light beam is focused by a lens onto the tip of two other fibers, one per channel, that transport the light to the outside of the spectrograph and inject it into the so-called exposure meter. The exposure meter produces a low-resolution spectrum and records the light entering the spectrograph in blue, green, and red channels for the two spectrograph-fiber channels independently. The exposure meter allows us to not only monitor the efficiency of the instrument, but also record the flux of the astronomical target and, as such, precisely compute the flux-weighted mean time of exposure (MTE); this, in turn, is used to determine the precise value of the Earth's barycentric velocity along the line of sight for which the recorded spectrum must be corrected for (Landoni et al. 2014).

\subsubsection{Ensuring instrumental stability}

The spectrograph is enclosed in a vacuum vessel that is in turn located inside a thermally controlled room. This environment offers stable conditions in terms of temperature and air pressure, minimizing their impact on the stability of the instrument (Álvarez et al. 2018). The spectrograph mechanics are designed and manufactured for the best possible metrological stability

\footnotetext{
2 www.eso.org/observing/etc/bin/gen/form?INS. NAME= ESPRESSO+INS. MODE=spectro
}

(Tenegi et al. 2016; Santana Tschudi et al. 2014). Materials are matched as much as possible for the thermal expansions coefficients, the setup is symmetric relative to a vertical plane (and thus gravity), no alignment mechanisms are available (alignment only by manufacturing and shimming), and no movable components exist inside the vacuum vessel; in other words, nothing can move along the entire light path after the optical fibers that feed the spectrograph. As for the FE, a fully mechanical alignment technique was developed and adopted during integration in Europe and Chile (Pariani et al. 2018; Genoni et al. 2016).

ESPRESSO is designed for an extreme RV precision of $10 \mathrm{~cm} \mathrm{~s}^{-1}$, which requires a perfect control of the metrological stability of the spectrograph. This extreme stability, which corresponds to a physical shift of the spectrum in the focal plane of the spectrograph by a few nanometers, cannot be achieved passively, and the physical parameters ruling it must be permanently controlled, or at least monitored. ESPRESSO therefore applies the "simultaneous-reference" technique (Mayor et al. 2003; Pepe et al. 2002), where the second fiber, B, can be fed with a spectral reference instead of the sky light and is used to track possible residual instrumental drifts down to the requested $\mathrm{RV}$ precision level. The simultaneous reference is only needed when the (photonic) RV error achieved on the scientific target is expected to be comparable to or lower than the typical instrumental drifts on the order of $\mathrm{m} \mathrm{s}^{-1}$. Consequently, the simultaneous-reference technique is applied for bright objects and when high RV precision is aimed at. For faint targets, the second fiber is fed with sky light so that sky background can be measured and subtracted.

It must be noted, however, that the desired (long-term) RV repeatability of $10 \mathrm{~cm} \mathrm{~s}^{-1}$ relies on the concept of repeatable wavelength calibration, and thus on the availability of an absolute spectral reference of wide spectral range. This spectral reference was supposed to be provided by the LFC, which, however, was still not operational at the time of writing this paper. For these reasons, the instrument was adapted to the combined use of a ThAr lamp and the Fabry-Pérot etalon, providing accuracy and precision, respectively, to a satisfactory level.

\subsection{Observing with ESPRESSO}

\subsubsection{Operations}

The operational flexibility of being able to feed light from any UT into ESPRESSO is used to maximize the overall scientific output of both the instrument and the observatory. In spite of the large number of subsystems and optical components involved, ESPRESSO observations can be triggered and started in any UT in less than ten minutes. The operators can thus optimize telescope time by selecting the UT where the observing pressure is the lowest at a given moment. Information from the used telescope, along with several environmental parameters, is stored in the night reports and in the FITS headers of the collected data.

An observation sequence consists of an acquisition and an observation part. The acquisition defines the observing mode and configures the hardware accordingly. The CS sets up the configuration of the chosen instrument mode, namely, the selection of the fiber head, exposure type, binning pattern, readout speed, and simultaneous reference. Meanwhile, the telescope is moved into position, and the target is acquired and positioned over the fiber (see the acquisition panel in Fig. 5, top, and the field image in Fig. 2). At the end of the acquisition, and throughout the full length of the observation, a secondary field-stabilization loop is 
Table 2. ESPRESSO's spectral format.

\begin{tabular}{|c|c|c|c|c|c|c|c|c|}
\hline PDO \# & $\begin{array}{l}\mathrm{CW} \\
{[\mathrm{nm}]}\end{array}$ & $\begin{array}{l}\text { FSR } \\
{[\mathrm{nm}]}\end{array}$ & $\begin{array}{l}\text { FSR start } \\
{[\mathrm{nm}]}\end{array}$ & $\begin{array}{l}\text { FSR end } \\
{[\mathrm{nm}]}\end{array}$ & $\begin{array}{l}\text { Order start } \\
{[\mathrm{nm}]}\end{array}$ & $\begin{array}{l}\text { Order end } \\
{[\mathrm{nm}]}\end{array}$ & $\begin{array}{l}\text { TSR } \\
{[\mathrm{nm}]}\end{array}$ & $\begin{array}{l}\text { Res. bin } \\
{[\mathrm{nm}]}\end{array}$ \\
\hline \multicolumn{9}{|c|}{ Red end of red arm } \\
\hline 78 & 784.45 & 10.06 & 779.45 & 789.51 & 778.98 & 790.64 & 11.66 & 0.00129 \\
\hline 79 & 774.52 & 9.80 & 769.65 & 779.45 & 769.11 & 780.65 & 11.54 & 0.00128 \\
\hline 87 & 703.30 & 8.08 & 699.28 & 707.37 & 698.34 & 708.89 & 10.56 & 0.00116 \\
\hline 88 & 695.31 & 7.90 & 691.38 & 699.28 & 690.40 & 700.84 & 10.45 & 0.00115 \\
\hline 101 & 605.81 & 6.00 & 602.83 & 608.83 & 601.50 & 610.66 & 9.16 & 0.00100 \\
\hline 102 & 599.87 & 5.88 & 596.95 & 602.83 & 595.60 & 604.67 & 9.07 & 0.00099 \\
\hline 111 & 551.24 & 4.97 & 548.76 & 553.73 & 547.30 & 555.65 & 8.36 & 0.00091 \\
\hline 112 & 546.31 & 4.88 & 543.89 & 548.76 & 542.41 & 550.69 & 8.29 & 0.00090 \\
\hline 116 & 527.48 & 4.55 & 525.21 & 529.76 & 523.70 & 531.71 & 8.01 & 0.00087 \\
\hline 117 & 522.97 & 4.47 & 520.74 & 525.21 & 519.22 & 527.16 & 7.94 & 0.00086 \\
\hline \multicolumn{9}{|c|}{ Blue end of red arm } \\
\hline \multicolumn{9}{|c|}{ Red end of blue arm } \\
\hline 117 & 522.97 & 4.47 & 520.74 & 525.21 & 519.13 & 527.03 & 7.89 & 0.00086 \\
\hline 118 & 518.54 & 4.39 & 516.35 & 520.74 & 514.72 & 522.57 & 7.84 & 0.00086 \\
\hline 135 & 453.24 & 3.36 & 451.57 & 454.92 & 449.83 & 456.82 & 6.99 & 0.00075 \\
\hline 136 & 449.91 & 3.31 & 448.26 & 451.57 & 446.52 & 453.46 & 6.94 & 0.00074 \\
\hline 152 & 402.55 & 2.65 & 401.23 & 403.88 & 399.48 & 405.75 & 6.26 & 0.00067 \\
\hline 153 & 399.92 & 2.61 & 398.61 & 401.23 & 396.87 & 403.10 & 6.22 & 0.00066 \\
\hline 160 & 382.42 & 2.39 & 381.23 & 383.62 & 379.50 & 385.47 & 5.96 & 0.00063 \\
\hline 161 & 380.04 & 2.36 & 378.87 & 381.23 & 377.15 & 383.07 & 5.93 & 0.00063 \\
\hline \multicolumn{9}{|c|}{ Blue end of blue arm } \\
\hline
\end{tabular}

Notes. For some representative diffraction orders of the echelle spectrograph, we provide the central wavelength (CW) in the middle of the order, the start and end wavelength of the FSR, and the start and end wavelength of the total (extracted) spectral range (TSR). Additionally, the spectral size of a physical detector pixel (resolution bin) located in the center of the order is given. PDO Physical Diffraction Order; CW Central Wavelength; [F/T]SR [Free/Total]-Spectral Range.

activated, keeping the target well centered in the fiber by moving the tip-tilt mirror (Landoni et al. 2016). A view of the secondaryguiding panel can be seen in the lower part of Fig. 5. The maximum correction frequency is $10 \mathrm{~Hz}$, but the actual speed depends on the magnitude of the target. The secondary-guiding system also provides pupil stabilization. However, given the excellent stability of the pupil, this correction is not performed during observations. The observation consists of one or more exposures (integrations) on the same target, ending in the readout and storage of the raw individual frames. The observation is assisted by the exposure meter, which measures the flux entering the fiber in real time and computes the flux-weighted MTE.

Operation in the 4-UT configuration is very similar to that in the 1-UT configuration. While in the 1-UT configuration the observing software dispatches instructions to the selected UT and ESPRESSO, in the 4-UT configuration it simultaneously sends instructions to the four UTs as well as the instrument. Presetting and target acquisition is done on all four UTs simultaneously. After this step, one needs to center the target of every UT on the specific fiber located inside the corresponding FE unit. This step is performed sequentially, moving the star's position on the focal plane of the fiber by offsetting the telescope. Once the target is centered and the secondary guiding is activated and stable for every telescope, the exposure is started. During the exposure, all four FE units, including the respective ADCs and secondary guiding, are active and independent. A screenshot of the field-stabilization panel in the 4-UT configuration is shown in Fig. 6.

Within seconds after the end of the exposure, the raw data are acquired and shown by ESO's real-time display (RTD) tool on the instrument machine (Fig. 7). The raw data are available for download through the ESO archive ${ }^{3}$. The ESPRESSO pipeline installed in Paranal automatically reduces the acquired data to provide a quick-look and a general check of the basic properties of the data and operational status of the instrument. Updated information on how to use ESPRESSO and the latest news on the instrument are available in the ESPRESSO User Manual ${ }^{4}$ and the dedicated News pages ${ }^{5}$ on the ESO website.

\subsubsection{Optimizing observations}

The main instrument characteristics in the various observing configurations and instrument modes are summarized in Table 3.

\footnotetext{
3 www.eso.org/archive/

4 wWw.eso.org/sci/facilities/paranal/instruments/ espresso/doc.html

5 www.eso.org/sci/facilities/paranal/instruments/ espresso/news.html
} 


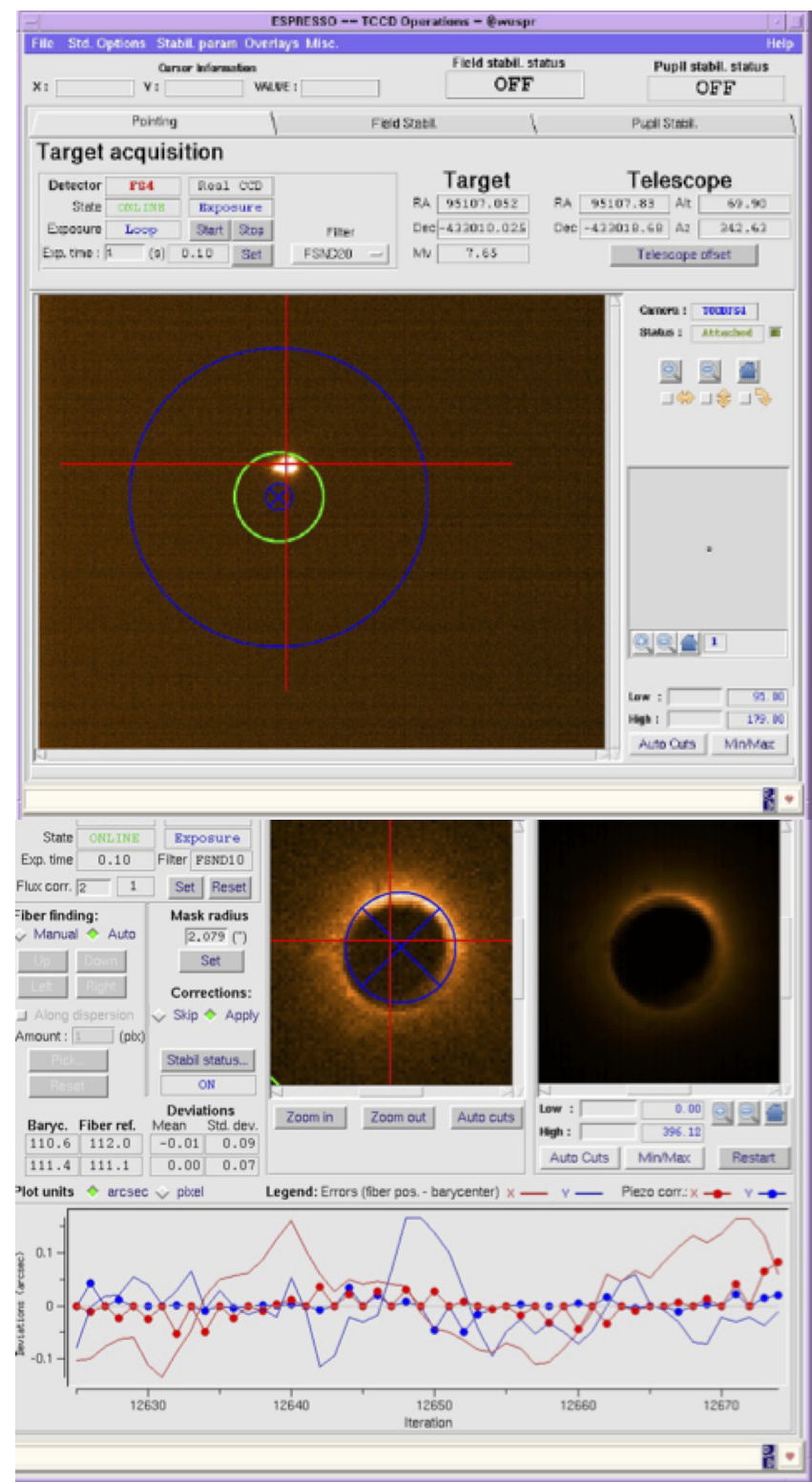

Fig. 5. Top: acquisition field in the 1-UT configuration. Bottom: secondary-guiding panel (field stabilization) during the scientific exposure. The observed target is centered on the fiber tip.

An ESPRESSO user can choose from two observing modes: the 1-UT configuration (one single telescope, any of the UTs) or the 4-UT configuration ${ }^{6}$ (all UTs simultaneously). In the 1-UT configuration, four different instrument modes exist. In HR mode, the light is collected using (octagonal) fibers with projected sky diameters of $1^{\prime \prime}$. This mode is intended for spectroscopy and RV monitoring. Three different detector readout modes offer optimized solutions depending on the target magnitude. In case of bright targets (HR11, $1 \times 1$ binning), the detector will be saturated quickly and the exposure time therefore has to be short. Overheads are reduced by a faster readout and the dynamic range is maximized by avoiding binning. Given the high signal level expected for a bright star, the higher RON is acceptable. For faint or very faint objects, the HR21 $(2 \times 1$

6 Combinations with two or three telescopes (degraded mode) are technically possible but not formally offered.

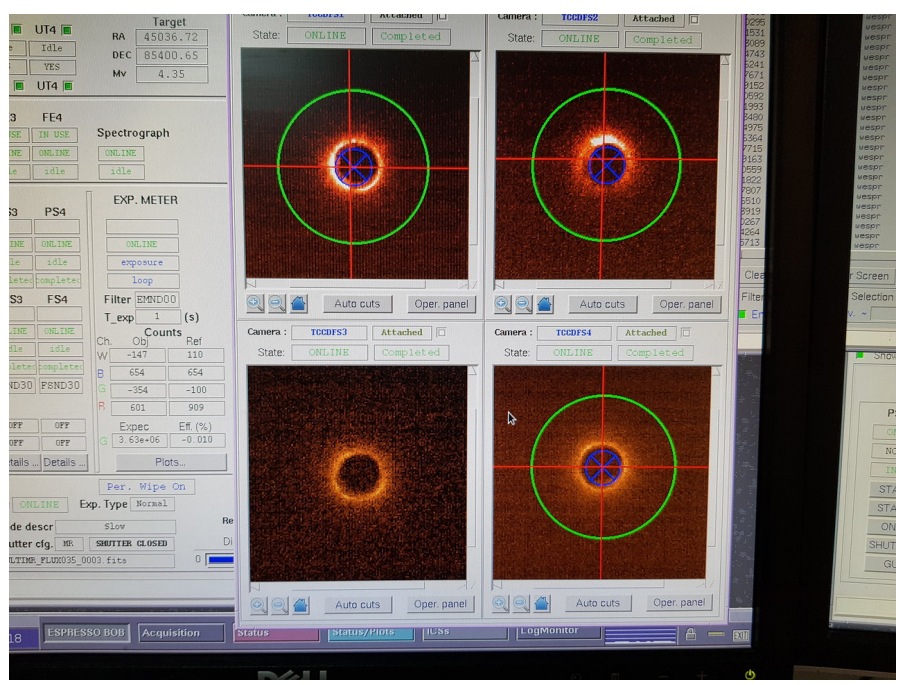

Fig. 6. Screenshot of the field-stabilization panel in the 4-UT configuration.

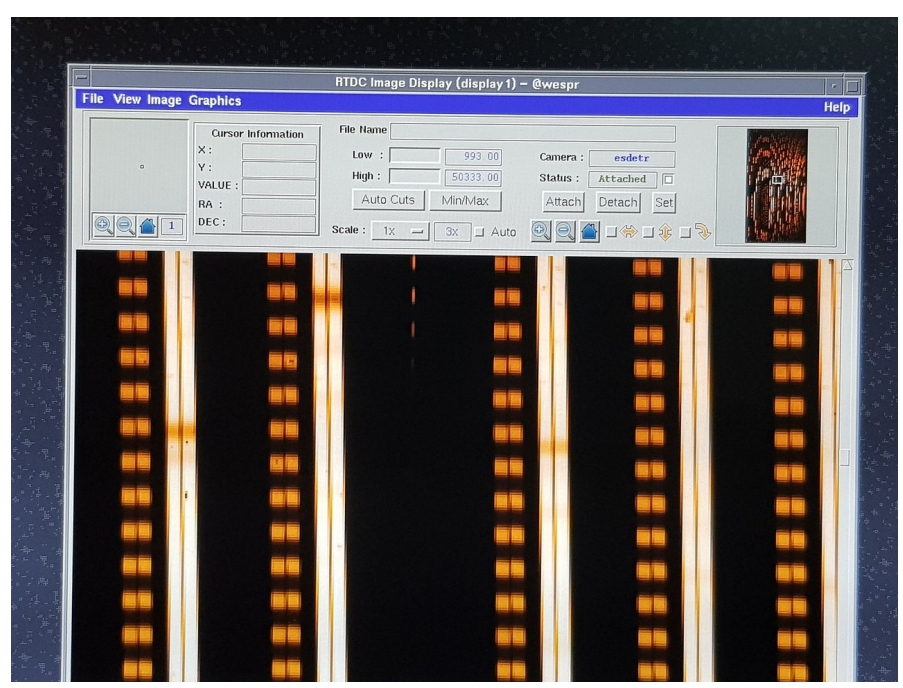

Fig. 7. Real-time display of a raw spectrum. A small portion of a star + Fabry-Pérot spectrum acquired in the 4-UT configuration is shown. The main dispersion is vertical. Each order consists of two spectral traces (slices) of the Fabry-Pérot, with its characteristic equidistant spectral lines, and two traces of the stellar spectrum, which essentially plots the continuum with a few absorption features. This figure is best understood when tilted by $90^{\circ}$ and compared to Fig. 4 .

binning) or HR42 ( $4 \times 2$ binning) should be used instead. The difference between these two modes is that the total RON per spectral bin is further reduced in the HR42 mode, and the limiting magnitude, at which the noise starts to be dominated by the detector RON, is improved. This gain comes at the cost of a slightly reduced resolving power since the resulting pixel sampling gets close to the Nyquist limit. This is especially the case on the blue side of the longest (reddest) echelle orders, where the anamorphic de-magnification reduces the size of the projected slit (fiber entrance) and thus the sampling. At the very blue end of the reddest echelle order, the sampling of the HR42 mode (worst case) is reduced by $30 \%$ (i.e., to about 1.7 pixels). Finally, the UHR11 mode delivers the highest resolving power, but the required use of a $0.5^{\prime \prime}$ diameter fiber leads to reduced spectral sampling and higher slit losses. Due to the latter issue, 
this mode is particularly suitable for ultra-high-resolution observations on relatively bright targets.

It must be noted that the pixel sampling given in Table 3 for the individual modes refers to the value expected at the center of an extracted echelle order. This value remains constant across all orders and over both detectors, as does also the resolution bin (pixel) size expressed in $\mathrm{m} \mathrm{s}^{-1}$, which results from a typical feature of cross-dispersed echelle spectrographs. Another very elegant characteristic of echelle spectrographs, when used in Littrow condition, is that the resolution bin size (again expressed in $\mathrm{m} \mathrm{s}^{-1}$ or $\left.\Delta \lambda / \lambda\right)$ remains essentially constant along the echelle order since the anamorphic effect on the projected slit (fiber) size and the variation of linear dispersion compensate almost perfectly. Finally, our design choice of oversizing the detector in order to extract at least $20 \%$ more than the FSR of the longest (reddest) orders (see Fig. 4) leads to the third elegant feature of echelle spectrographs: by summing up the flux of a given wavelength recorded at the edges of two subsequent echelle orders, one recovers almost the same number of photoelectrons as for wavelengths located close to the blaze (center) of the echelle order. This means that after extracting and merging the orders, the detection efficiency obtained at a given wavelength is close to constant. For all these reasons, we will describe hereafter the measured signal (and $\mathrm{S} / \mathrm{N}$ ) as the value obtained at the center of the order and for a given extracted pixel (resolution bin), under the motivated assumption that this value varies only by a small amount along the echelle order.

By design, the 4-UT observing configuration is intended for spectroscopy of very faint targets. The lower resolution is a price to pay for the usage of an equivalently wider (square) fiber in the spectrograph that can carry the light of the four telescopes. Collecting the light from the four telescopes reduces the contribution of detector readout and (partially) dark noise when adequate slit configurations and binning levels are adopted. The two instrument modes MR42 ( $4 \times 2$ binning) and MR84 $(8 \times 4$ binning) therefore enable very low $\mathrm{S} / \mathrm{N}$ spectroscopy and the study of fast transient events.

The observations are prepared using the usual $\mathrm{P} 2$ tool $^{7}$. On top of allowing the user to select from all the possible instrument configurations and defining observational constraints, it provides advanced functionalities such as target data retrieval from the SIMBAD database, definition of "blind offset" from nearby stars, and monitoring of the field scanned by the reference fiber when in simultaneous sky mode. For the computation of the exposure time and the expected $\mathrm{S} / \mathrm{N}$ and $\mathrm{RV}$ precision, the user is referred to the ESPRESSO ETC ${ }^{8}$. We shall nevertheless provide a benchmark: in a 1 min integration in HR11 on a K2 star with $V=8$ mag observed at an airmass of 1 and under median seeing conditions of $0.8^{\prime \prime}$, an $S / N \sim 115$ at blaze and per extracted pixel (or resolution bin) of $0.486 \mathrm{~km} \mathrm{~s}^{-1}$ is reached. This corresponds to a gain of about two magnitudes with respect to HARPS at the $3.6 \mathrm{~m}$ telescope. By using the relation between $\mathrm{S} / \mathrm{N}$ and $\mathrm{RV}$ precision derived for nonrotating $\mathrm{G}$ and $\mathrm{K}$ dwarfs, one estimates that this $\mathrm{S} / \mathrm{N}$ delivers a photon-noise limited $\mathrm{RV}$ precision of about $50 \mathrm{~cm} \mathrm{~s}^{-1}$. Further details are available in Sect. 4.1.4.

An option available to the observer is the use of fiber B. ESPRESSO provides two mutually exclusive possibilities: The user can either illuminate fiber B with a simultaneous spectral reference to measure the instrument's internal drift or to use it to independently measure the sky background. In the first option,

\footnotetext{
7 www.eso.org/sci/observing/phase2/p2intro.html

8 wWw . eso.org/observing/etc/bin/gen/form?INS . NAME= ESPRESSO+INS. MODE=spectro
}

the secondary fiber is illuminated by the Fabry-Pérot light. The many spectral lines are used to measure and correct for the minute-scale motions of the spectra on the CCDs induced by the short-term variations of pressure and temperature inside the spectrograph and the detector. This option becomes important when one wants to achieve an RV precision similar to or better than the intrinsic stability of the instrument over the timescale of a night of about $1 \mathrm{~m} \mathrm{~s}^{-1} \mathrm{rms}$. If one aims at on-target RV photon-noise precision better than this value, then it is recommended to use the simultaneous reference. Conversely, if this precision is not required, or one wants to perform any kind of measurement for which sub- $\mathrm{m} \mathrm{s}^{-1}$ precision is not needed (e.g., transmission spectroscopy), then the use of the simultaneous reference is not necessary. In fact, in the latter case, and when high spectral fidelity observations are desired, one needs instead to consider the contribution of the sky background. During solar activity minima and in a region of the sky devoid of starlight or moonlight, the $V$-band magnitude of sky spectra as seen through a $1^{\prime \prime}$ fiber is still of $V=20-21 \mathrm{mag}$. In the presence of a full Moon and thin clouds, the sky brightness can increase by many magnitudes. If we consider this, then it becomes clear that moonlight contamination may become an issue for precise RV measurements even at magnitudes as low as $V \leq 11 \mathrm{mag}$ (see e.g., Cunha et al. 2013 or Roy et al. 2020). It is therefore recommended to use fiber B to measure the sky background for "faint" targets. The numbers and magnitudes provided here are only a general guideline. A more detailed optimization should be made for each program as a function of the specific science case.

\subsection{Data flow}

\subsubsection{Concept and elements}

For instruments installed at the VLT, the concept of a data flow system (DFS) denotes a closed-loop software system that incorporates various software packages able to track the flow of scientific data with the highest possible level of quality. Its logical path follows the typical life-cycle of an astronomical observation: from the proposal preparation and handling, through observation preparation, instrument operation, and control, to science data archive, reduction, and quality control. Compared with other spectrographs hosted by ESO, the ESPRESSO DFS has some peculiarities.

The ESPRESSO DFS concept (Di Marcantonio et al. 2018) was conceived during its preliminary design phases with the goal of maximizing operational efficiency, flexibility, and scientific output while complying with the standard Paranal Observatory operational scheme. The main challenge derives from the requirement to operate ESPRESSO in a seamless way with any of the UT's or with all four UT's simultaneously. This must be possible not only with a predetermined schedule, but also "on the fly". The flexibility in ESPRESSO's operations has been tackled by adopting a new DFS deployment plan described in Di Marcantonio et al. (2018) that is exceptional under various aspects because it has to cope with various telescope and instrument configurations while remaining operationally simple. Figure 8 shows the main ESPRESSO DFS elements and their final deployment. Besides the software packages already described, part of the software for the control of the CT devices has been incorporated into the VLT telescope CS to allow CT operations even when ESPRESSO is offline (thus avoiding conflicts, e.g., with instruments of the VLT Interferometer operations). In addition to the standard DFS software packages, ESPRESSO is the first instrument to also provide a data analysis 
Table 3. ESPRESSO's instrument modes and characteristics.

\begin{tabular}{|c|c|c|c|c|c|c|}
\hline \multirow{2}{*}{$\begin{array}{l}\text { Observing configuration } \\
\text { Instrument mode }\end{array}$} & \multicolumn{4}{|c|}{ 1-UT } & \multicolumn{2}{|c|}{ 4-UT } \\
\hline & UHR11 & HR11 & HR21 & HR42 & MR42 & MR84 \\
\hline Simultaneous reference & \multicolumn{6}{|c|}{ Available (no simultaneous sky background on fiber B) } \\
\hline Sky subtraction & \multicolumn{6}{|c|}{ Available (no simultaneous reference on fiber B) } \\
\hline Wavelength range $[\mathrm{nm}]$ & \multicolumn{6}{|c|}{$378.2-788.7$} \\
\hline Median resolving power $R=\lambda / \Delta \lambda$ & $>190000^{(a)}$ & 138000 & 138000 & 130000 & 72500 & 70000 \\
\hline Aperture on sky ["] & 0.5 dia. & 1.0 dia. & 1.0 dia. & 1.0 dia. & $4 \times 1.0$ dia & $4 \times 1.0$ dia. \\
\hline Spectral sampling [physical pixels] & 2.5 & 4.5 & 4.5 & 4.5 & 10 & 10 \\
\hline Spatial sampling [slices $\times$ physical pixels] & $2 \times 5$ & $2 \times 9$ & $2 \times 9$ & $2 \times 9$ & $2 \times 20$ & $2 \times 20$ \\
\hline Detector readout speed $\left[\mathrm{kpix} \mathrm{s}^{-1}\right]$ & 300 & 300 & 100 & 100 & 100 & 100 \\
\hline Detector binning, spatial × spectral [pixels] & $1 \times 1$ & $1 \times 1$ & $2 \times 1$ & $4 \times 2$ & $4 \times 2$ & $8 \times 4$ \\
\hline Effective spectral sampling [extracted pixels] & 2.5 & 4.5 & 4.5 & 2.25 & 5 & 2.5 \\
\hline Detector readout noise $(\mathrm{RON}) \mathrm{red} / \mathrm{blue}{ }^{(b)}\left[\mathrm{e}^{-}\right]$ & $5 / 8$ & $5 / 8$ & $2 / 3$ & $2 / 4$ & $2 / 4$ & $2 / 5$ \\
\hline Detector conversion factor red/blue [ $\left.\mathrm{e}^{-} / \mathrm{ADU}\right]$ & $1.15 / 1.1$ & $1.15 / 1.1$ & $1.13 / 1.09$ & $1.13 / 1.09$ & $1.13 / 1.09$ & $1.13 / 1.09$ \\
\hline Total peak efficiency & $5 \%$ & $10 \%$ & $10 \%$ & $10 \%$ & $10 \%$ & $10 \%$ \\
\hline
\end{tabular}

Notes. ${ }^{(a)}$ To be considered as a lower limit since many thorium lines used for the analysis are partially resolved at this resolution. ${ }^{(b)}$ The "FAST" readout modes (HR11 and UHR11) of the blue detector are still undergoing optimization.

package that is able to extract relevant astronomical observables from the reduced data. The following DFS subsystems are specific to ESPRESSO: (1) the ETC hosted on the ESO web page $^{9}$; (2) the CS with the full suite of acquisition, observation, and calibration templates that are able to control all vital parts of the instrument and the CT (Calderone et al. 2018); (3) the data reduction software (DRS) package (or "pipeline") capable of providing "science-ready" reduced data only minutes after the end of the individual observation; (4) the data analysis software (DAS) package that produces higher-level astronomical observables with no or limited supervision; (5) the DRS and DAS are distributed to the community ${ }^{10}$.

The DRS and DAS packages are delivered in an integrated way even though both can be employed independently. In standard operation, the DRS will be automatically triggered once raw FITS files are produced by the ESPRESSO CS. In visitor mode, both the DRS and DAS can be used offline and on a stand-alone basis.

\subsubsection{Data reduction software}

The ESPRESSO DRS, referred to as pipeline, is a scientific software package whose purpose is to process the raw FITS frames generated by the instrument into reduced data products of various kinds, including science-grade high-resolution spectra of any astrophysical sources observed by ESPRESSO. An in-depth description of the ESPRESSO DRS is beyond the scope of this paper and will be provided elsewhere (Lovis et al., in prep.). We limit ourselves here to a general overview.

The ESPRESSO DRS builds on a vast heritage of data reduction techniques and software. On the scientific side, it represents the next generation of a series of pipelines that started with the ELODIE spectrograph in the early 1990s (Baranne et al. 1996), then followed by CORALIE (Queloz et al. 2000; Pepe et al. 2002), HARPS (Mayor et al. 2003), and finally HARPS-N (Cosentino et al. 2012). Many of the data reduction steps and algorithms implemented in the ESPRESSO pipeline find their

\footnotetext{
9 wwW.eso.org/observing/etc/bin/gen/form?INS. NAME= ESPRESSO+INS. MODE=spectro

10 wWW . eso.org/sci/software/pipelines/index.html
}

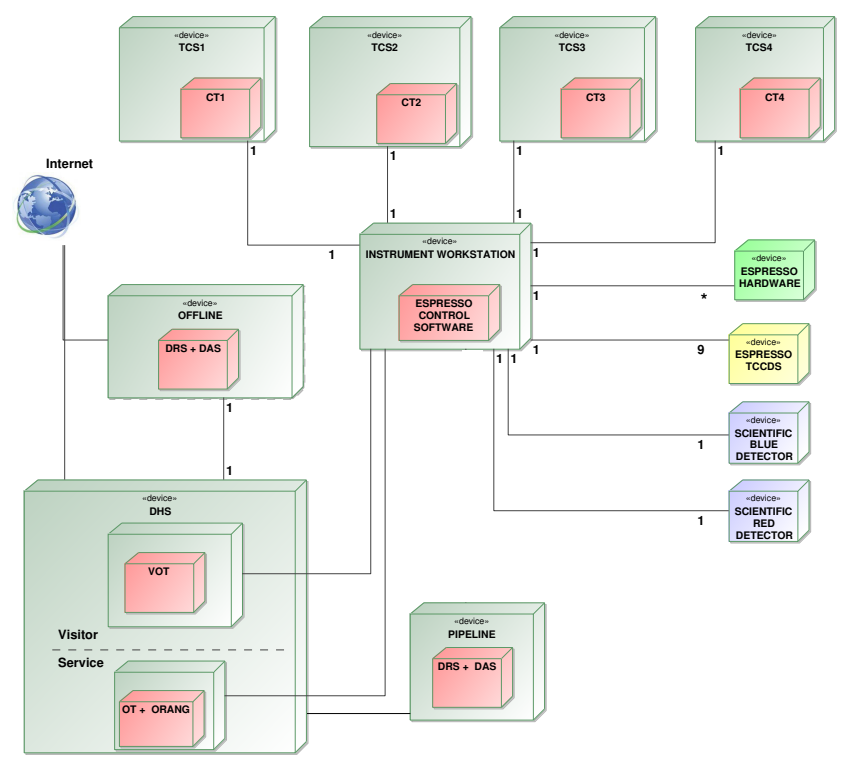

Fig. 8. ESPRESSO final DFS deployment at Paranal. This figure is from Pepe et al. (2014) and ESPRESSO Project documentation.

origins in the HARPS pipeline. However, several steps were significantly re-designed and improved with respect to HARPS to meet the more challenging requirements of ESPRESSO.

On the implementation side, the ESPRESSO pipeline is based on a software framework imposed by ESO on all VLT instrument pipelines, namely the Common Pipeline Library (CPL), a C-based toolkit offering basic functionalities for astronomical data manipulation and reduction ${ }^{11}$. The pipeline is structured as a set of top-level recipes, written in C, which handle the various raw data types produced by the instrument (all types of calibration and science frames). The DRS recipes can be called either through the EsoRex command-line interface or through the EsoReflex graphical user interface (GUI) workflow environment, both of which are developed and maintained by ESO. The ESPRESSO pipeline, including its source code and

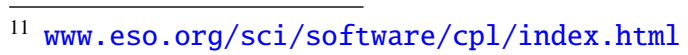


the DRS manual, is publicly available from the ESO pipeline repository ${ }^{12}$.

Data processing is organized as a sequential reduction cascade in which each raw data type is associated with a dedicated DRS recipe. The pipeline generates intermediate data products at each stage, many of which are then used as inputs in the subsequent steps of the cascade. The final task is the reduction of science spectra. The main steps of the science processing are: (1) bias and dark subtraction; (2) subtraction of inter-order background; (3) optimal extraction of spectral orders using master flat-field as order profile, with masking of dead, hot, and saturated pixels and rejection of cosmic rays; (4) creation of extracted spectra (S2D) in (order, pixel) space with associated error and quality maps; (5) flat-fielding and de-blazing of S2D spectra; (6) wavelength calibration of S2D spectra using either a combination of ThAr and Fabry-Perot sources or the LFC; 97 computation of barycentric correction and shift of wavelength solutions to the Solar System barycenter; (8) instrumental drift computation and correction using fiber B (simultaneous reference mode); (9) extraction of sky spectrum and creation of sky-subtracted S2D spectra using fiber B (simultaneous sky mode); (10) creation of rebinned and merged 1D spectra (S1D) with associated error and quality maps; (11) creation of fluxcalibrated S1D spectra using estimated absolute efficiency and extinction curves; (12) computation of the cross-correlation function $(\mathrm{CCF})$ of the S2D spectrum with a stellar binary mask; (13) fit of the CCF with a Gaussian model to derive RV and associated CCF parameters (full width at half maximum; FWHM, contrast, bisector span, asymmetry); and (14) computation of sky-subtracted CCFs with associated RV and CCF parameters (simultaneous sky mode).

The ESPRESSO DRS has a modular design and can be adapted to the different modes of ESPRESSO, as well as to other echelle spectrographs like HARPS, through a set of configuration files. The DRS recipes themselves are the same for the different modes and instruments.

\subsubsection{Data analysis software}

The ESPRESSO DAS is designed to perform scientific measurements on reduced data and to provide users with high-level science-grade observables and products, such as lists of lines, continuum models, and best-fit values of physical parameters. Like the DRS, it is structured as a set of recipes that can be launched individually (using the ESO's command-line interface EsoRex) or collectively as a workflow (through the ESO Reflex environment). Each recipe performs a specific operation and may take as input both DRS products and static calibration (list of reference lines, etc.).

To cope with the nonlinear nature of the analysis procedure, six different Reflex workflows are included in the DAS, each with its own specific cascade of recipes. Three workflows are devoted to the analysis of stellar spectra. First, star_I combines multiple exposures of the same stellar spectrum correcting for RV (recipe espda_coadd_spec), determines the equivalent width of selected absorption lines (recipe espda_compu_eqwidth), and compares the equivalent widths with static calibrations to determine the effective temperature and the metallicity of the star (recipe espda_compu_starpar).

\footnotetext{
12 www.eso.org/sci/software/pipelines/espresso/ espresso-pipe-recipes.html
}

Second, star_II combines multiple exposures of the same stellar spectrum without correcting for RV (recipe espda_coadd_spec), estimates the continuum level order by order (recipe espda_fit_starcont), generates a synthetic spectrum corresponding to a given set of stellar and broadening parameters (recipe espda_synth_spec), and computes and fits the CCF between the synthetic spectrum and the observed one to estimate the RV of the star (recipe espda_rv_synth) to finally compare the observed spectrum with the synthetic spectrum. As an example of the star_II workflow, we display a normalized spectrum of $\tau$ Cet, computed by co-adding a subsample of 40 ESPRESSO HR11 2D-spectra, in Fig. 9.

Third, star_III estimates and corrects for the RV by crosscorrelating the spectrum in the order-wavelength space with a predefined mask (recipe espda_compu_radvel) and measures the integrated fluxes in given passbands to estimate the stellar activity indices (recipe espda_compu_rhk).

Two workflows are devoted to the analysis of a quasi stellar object (QSO) spectra. First, qso_I combines multiple exposures of the same QSO spectrum (recipe espda_coadd_spec), lets the user mask the spectral regions that should not be considered in the analysis (recipe espda_mask_spec), detects the absorption lines (recipe espda_create_linelist), fits the continuum level by provisionally fitting and removing the absorption lines (recipe espda_fit_qsocont), and finally identifies the detected lines and combines them into absorption systems, namely different ionic transitions at the same redshift (recipe espda_iden_syst). Second, qso_II fits the identified lines with Voigt profiles (recipe espda_fit_line) and revises the continuum level taking into account the more precise line fitting (recipe espda_fit_qsocont).

Additionally, a coadd workflow is provided that includes only recipes espda_coadd_spec and espda_mask_spec. This is meant to be a fast tool to visualize the co-added spectrum and check the $\mathrm{S} / \mathrm{N}$.

The combination of several workflows allows for a flexible analysis while maintaining a large degree of automation in the procedure. Most recipes are equipped with interactive Python scripts (automatically called by Reflex) to plot and interact with the data, changing recipe parameters and prompting the re-execution of the recipe. All products are provided in FITS format and can be easily handled by other analysis tools.

Algorithmically, the DAS includes both long-tested solutions, like the line-finding technique implemented in ARES (Sousa et al. 2007), and brand-new methods, like the drizzlinglike approach to coaddition and the simultaneous continuumline fitting in QSO spectra (Cupani et al. 2016, 2019). The strict requirements imposed by the ESPRESSO science cases necessitated novel solutions that benefit the entire community and are evolving beyond the original scope of the instrument (see e.g., the Astrocook data analysis package Cupani et al. 2018 and the ARESv2 package Sousa et al. 2015).

\section{On-sky performance}

In the following sections, we will describe the on-sky performance of ESPRESSO, that is, the performance of the complete system (including the telescopes) in all its configurations and the observing modes offered to the users. We will address the toplevel technical requirements that determine the scientific capabilities of ESPRESSO. 

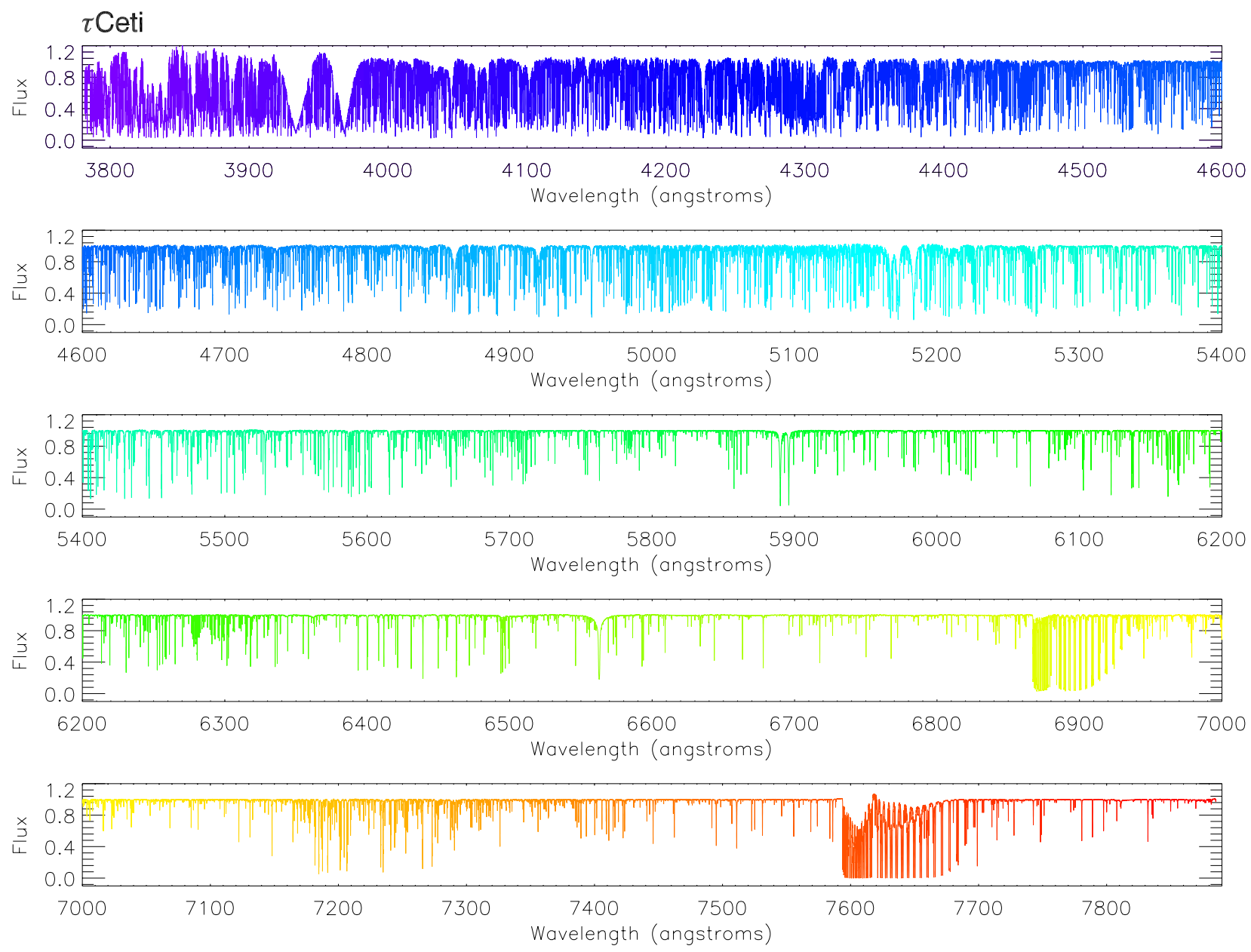

Fig. 9. Output of the star II workflow after normalizing and co-adding 40 HR 11 ESPRESSO spectra of $\tau$ Cet.

\subsection{Spectroscopic performance}

\subsubsection{Spectral range}

The wavelength range of ESPRESSO was originally specified by ESO to cover at least the full visible range from 380 to $680 \mathrm{~nm}$, similar to the HARPS spectrograph, with an optional extension to $350 \mathrm{~nm}$ on the blue side and to $760 \mathrm{~nm}$ on the red side. While the extension to the red side could easily be implemented, the blue extension would have produced considerable technical challenges. The implemented spectral range continuously covers the domain from $378.2 \mathrm{~nm}$ to $788.7 \mathrm{~nm}$. This coverage is provided for all instrument modes (HR, UHR, and MR), albeit at different resolving powers.

In order to illustrate the spectral range, richness, and quality, we show the extracted and wavelength-calibrated 1D-spectrum of $\tau$-Cet (HD 10700) as observed in the UHR mode in the top panel of Fig. 10. The lower two panels provide a zoom-in on the very blue and very red ends of the spectrum.

\subsubsection{Resolving power}

The resolving power $R=\lambda / \Delta \lambda$ of a spectrograph is defined as the ratio between the wavelength $\lambda$ and the FWHM $\Delta \lambda$ of a non-resolved spectral line. In astronomical spectrographs, the slit width or fiber diameter is the result of the trade-off between resolving power and slit-efficiency. In order to contain slit losses, the on-sky projected slit width (fiber diameter) is usually chosen to be comparable to or larger than the median seeing of the observing site. In opposition, the spectrograph's optical design (size, dispersion) is adapted to produce the desired resolving power. As a consequence, the resolving power is not determined by the diffraction limit of the optics, but simply by the fiber diameter. Transferred to ESPRESSO, this implies that the spectral resolution $\Delta \lambda$ covered by the fiber projected on the detector is directly proportional to the fiber width and that the resolving power is inversely proportional. The different modes of ESPRESSO produce, therefore, a different resolving power, as shown in Table 3.

However, the geometrical image of the optical fiber is convolved with the "local" instrumental profile (IP) of the spectrograph that represents the impact of optical aberrations and diffraction on the image of a perfectly monochromatic source feeding the spectrograph. As a consequence, the fiber image on the detector will be slightly blurred and the resolving power will be reduced. Of course, the effect is larger, in relative terms, when the IP is comparable in width to the fiber diameter. In other words, the narrower the fiber - and thus the higher the resolving power - the larger the reduction in resolving power will be compared to its theoretical value computed from geometrical considerations alone.

In order to study the effectively achieved resolving power in the various modes and across the full spectrum, we have used thorium-argon lamp calibration frames, assuming that most of the thorium emission lines are not or are only marginally 

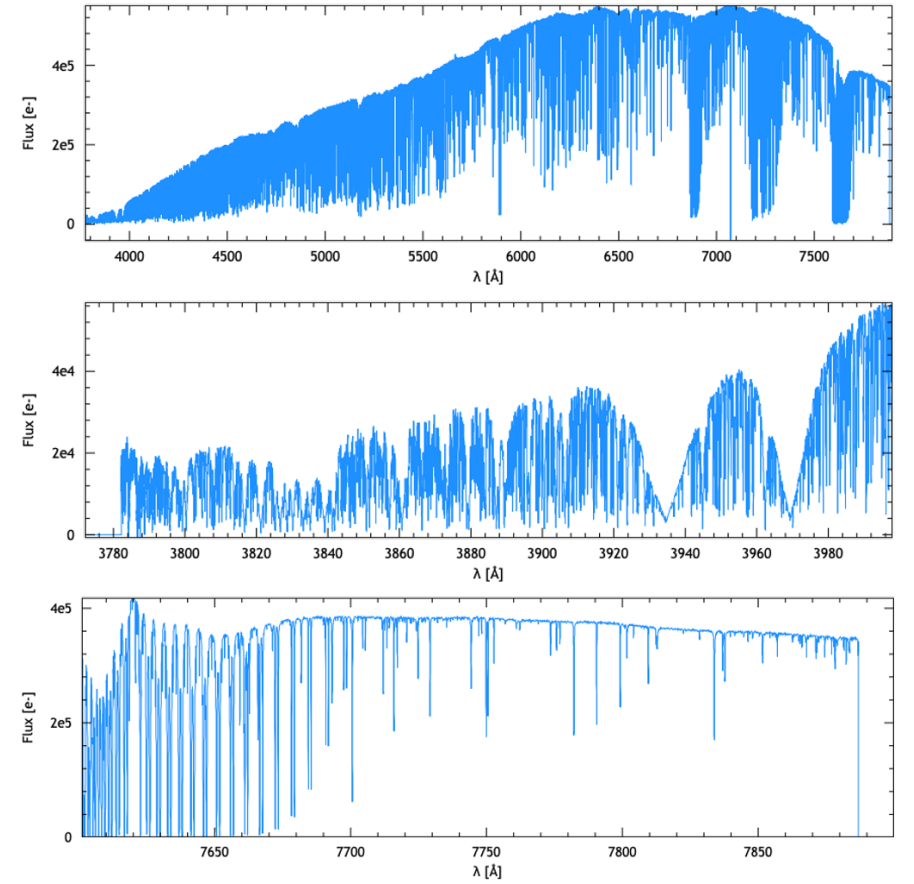

Fig. 10. Top panel: full extracted and wavelength calibrated spectrum of $\tau$-Cet observed with ESPRESSO in UHR mode. Middle panel: zoom-in on the blue end of the spectrum emphasizing the calcium doublet on the right side. Bottom panel: zoom-in on the red end of the spectrum showing the deep and narrow sequence of telluric absorption lines on the left side.

resolved ${ }^{13}$ by the spectrograph. For all non-saturated thorium lines with sufficient signal we measured the line width and converted the FWHM into resolving power. Using 2D interpolation, we produced a 2D map of the resolving power as a function of "extracted pixel" (spectral direction, i.e., main dispersion) and echelle order (spatial direction, i.e., cross-dispersion). The three images in Fig. 11 represent, from top to bottom, the UHR, the $\mathrm{HR}$, and the MR modes. Each image is composed of one larger frame (top) showing the red chip and a smaller frame (bottom) showing the blue chip. The wavelength increases from bottom to top (cross-dispersion direction) and from left to right (maindispersion direction along the order). For each order of the HR and UHR modes, both slices are shown side-by-side, and, as can be seen, the resolving power is slightly different. In the MR mode, the two (adjacent) slices must be extracted simultaneously by the DRS, resulting in a resolution map of one line per order. The map also shows the position of the thorium line used for determining the resolving power and interpolating the maps.

In the HR mode, the standard mode, a median resolving power of $R=138000$ is measured (requirements: $R=120000$ ). In the MR mode (4-UT configuration), the resolving power is reduced by about a factor of 2 (i.e., $R=72500$ ) due to the larger fiber diameter (requirement: $R=30000$ ). The UHR mode achieves a median resolving power on the order of $R>190000$ in the blue arm, while exceeding $R=220000$, and thus being up to specification, in the red arm.

At first glance, a similar global behavior is observed in all modes. The blue spectrum (lower chip) is slightly less well

\footnotetext{
${ }^{13}$ From measurements using the (unresolved) LFC lines, we actually know that most of the thorium lines are partially resolved. Since we cannot use the LFC over the full wavelength range, we prefer to provide the (safe) lower limits for the resolving power.
}
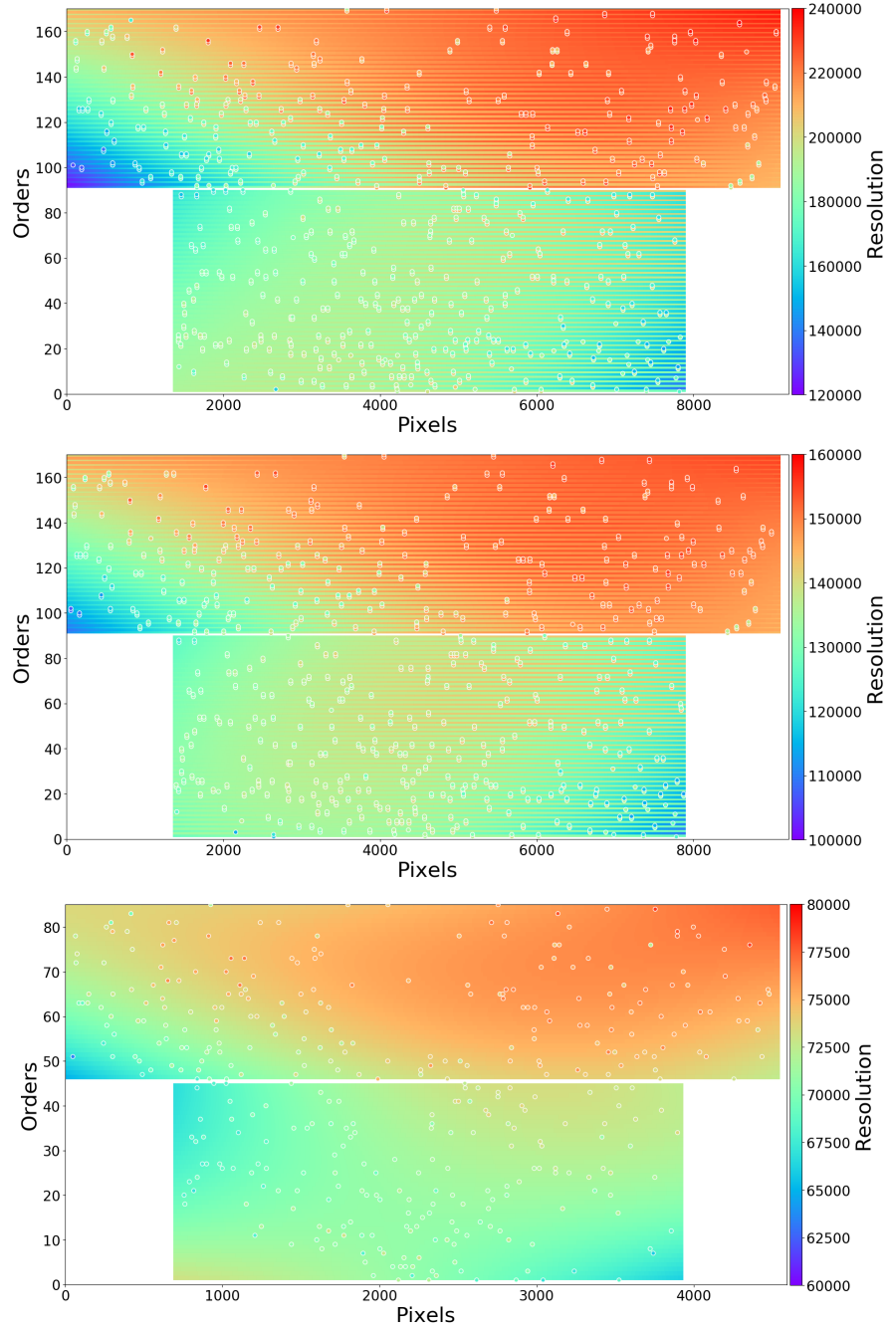

Fig. 11. From top to bottom: 2D maps of ESPRESSO's resolving power for its three instrument modes UHR, HR, and MR. For each mode, the two CCDs are represented (top = red chip, bottom = blue chip). The $y$-axis does not represent the physical diffraction order number but denotes a continuous (DRS-internal) numbering of every individually extracted slice of each order sorted by increasing wavelength $(1=$ bluest wavelengths). The white dots show the location of the thorium lines used for the computation of the resolving power. Since in the MR mode the two slices of a given order are merged together by the extraction algorithm, only half the number of orders are present. Their lines have been graphically increased in width to represent the same detector area as the two other modes.

resolved and a trend to higher resolving power is observed along every order from left (blue end) to right (red end). Slight variations of resolving power are indeed expected from optical-design considerations and are known to depend on the manufacturing parameters of each component and the effective alignment of the spectrograph.

The number of free parameters and manufacturing unknowns is so large that a detailed understanding of the observed map is close to impossible. For this reason, the spectrograph was aligned in such a way as to achieve a resolution map as homogeneous as possible while trying to maximize median resolving power. The following facts attest to the success of this strategy. First, the maps look very similar in all modes; this means that the impact of the image quality of the spectrograph is not the dominant factor in the measured resolving power. Second, the 


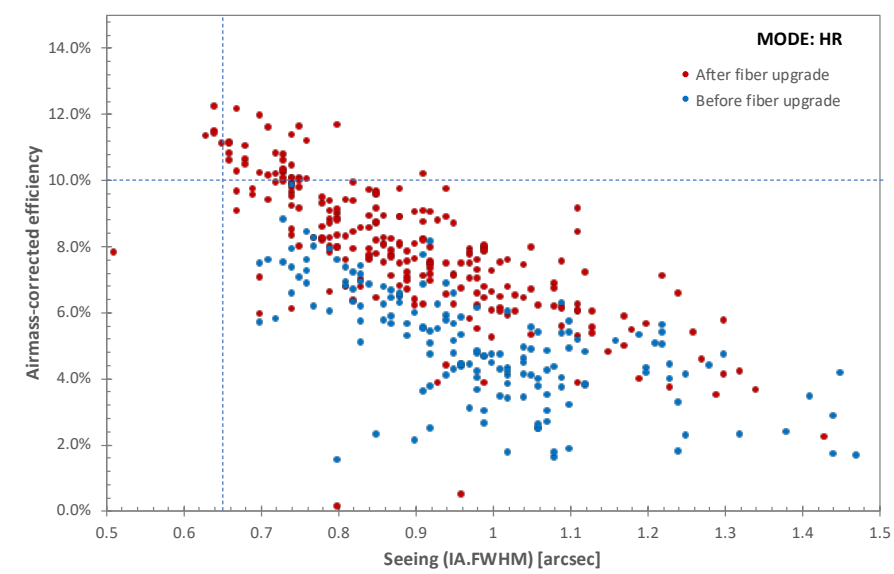

Fig. 12. Photon-detection efficiency at $550 \mathrm{~nm}$ and airmass 0 as a function of telescope seeing (image quality IA.FWHM) before (blue dots) and after (red dots) the fiber-upgrade mission.

resolving power in the MR and HR modes are both compliant with the original requirements. On the other hand, the resolving power in the UHR mode is not compliant with the original requirement, at least on the blue chip. It must be recalled, however, that the resolving power has been determined using thorium lines that are partially resolved in this mode. Finally, we note that ESPRESSO is today the only spectrograph in the Southern Hemisphere on an $8 \mathrm{~m}$ class telescope that delivers in a single exposure the entire visible spectrum at a resolving power of $R>190000$.

\subsubsection{Detection efficiency}

We define the (total) photon-detection efficiency as the ratio between the number of photoelectrons per wavelength bin at $\lambda=550 \mathrm{~nm}$ measured on the detector at the blaze of the echelle order, as well as the number of photons received from a pointlike source before entering the atmosphere assuming photometric conditions and observation airmass $A M=1$. Since in a spectrograph the throughput strongly depends on the seeing, ESPRESSO's requirements were specified such that a peak efficiency of $10 \%$ is reached at the center of the echelle order for a seeing of $0.65^{\prime \prime}$ as measured in the focal plane of the telescope.

Figure 12 shows the measured detection efficiency at $550 \mathrm{~nm}$ for airmass $A M=0$ (no absorption) as a function of measured image quality IA.FWHM ${ }^{14}$. The IA.FWHM represents the "seeing" value at the Nasmyth focus of the telescope as computed by ESO's standard "Image Analysis" tool. Blue data points show the measured efficiency of ESPRESSO shortly after first commissioning, while the red data points were obtained in the months following the fiber-upgrade mission of June 2019. The plot demonstrates that, after the fiber upgrade, the photondetection efficiency at airmass 0 and for a seeing of $0.65^{\prime \prime}$ is close to $12 \%$, about $40 \%$ higher than prior to the fiber exchange. Introducing an airmass of 1 causes the values to drop by $10-20 \%$ depending on the wavelength, such that the peak photon-detection efficiency still marginally reaches the specified $10 \%$ level.

\footnotetext{
${ }^{14}$ FITS-header keyword available in the raw images. IA.FWHM gives the FWHM measured on the individual sub-apertures of the ShackHartmann scaled to zenith and at $550 \mathrm{~nm}$. DIMM seeing is instead defined at zenith and $500 \mathrm{~nm}$.
}
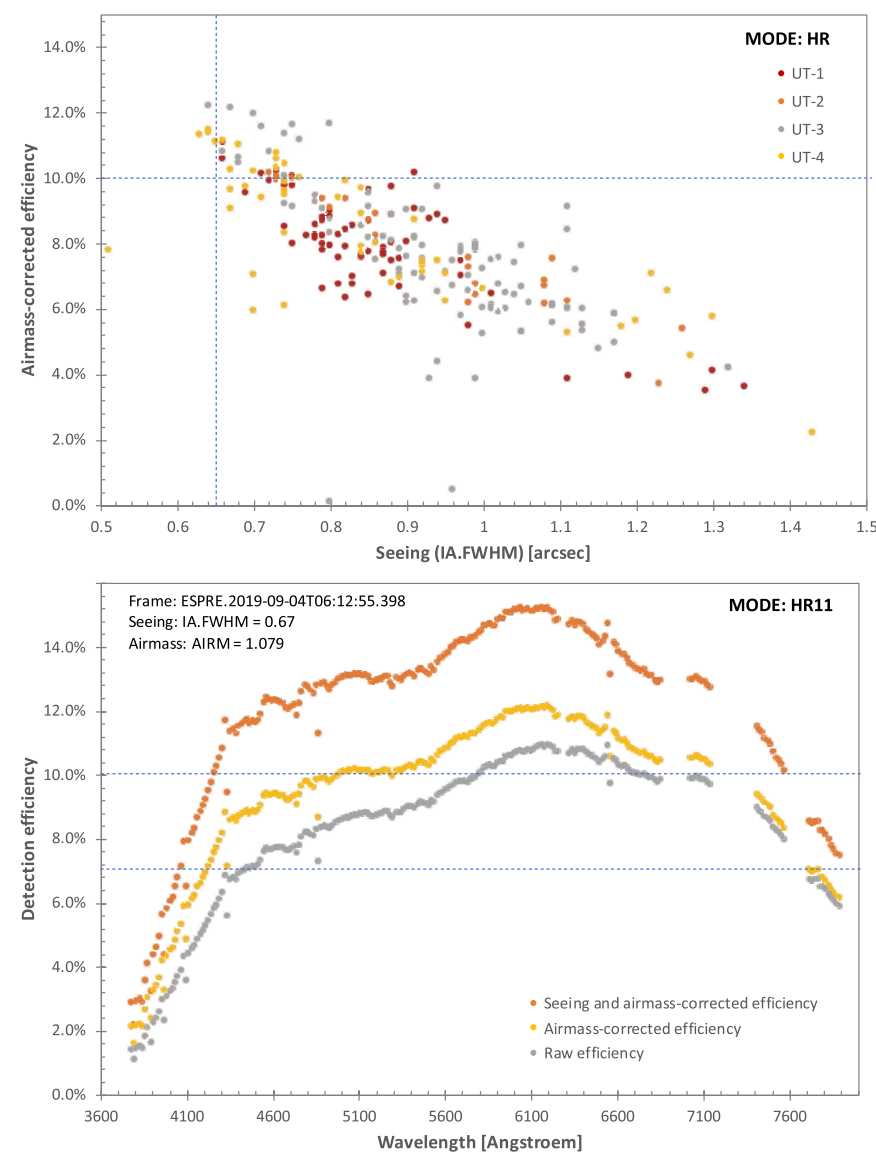

Fig. 13. Bottom: airmass-corrected efficiency (no atmospheric absorption) for the HR mode as a function of seeing (image quality $I A . F W H M$ ). The plot combines the measurements from all telescopes. Top: total photon-detection efficiency for the HR mode in binning $1 \times 1$ as a function of wavelength. An example of an image quality of IA.FWHM of 0.67 " and airmass of 1.079 is shown.

In the following, we present the photon-detection efficiency for all modes and as a function of wavelength, as obtained after the fiber upgrade. Figure 13 shows the detection efficiency for the HR mode as a function of wavelength as measured by the pipeline (raw), corrected for atmospheric absorption (airmasscorrected) and slit losses (seeing and airmass-corrected). The raw efficiency reaches $10 \%$ at $550 \mathrm{~nm}$ and exceeds it in the range between 550 and $680 \mathrm{~nm}$. The same plot shows, however, that the requirement that the photon-detection efficiency not drops below $7 \%$ inside the specified minimum wavelength range (380-680 nm) of ESPRESSO is not completely fulfilled. Indeed, for wavelengths bluewards of $430 \mathrm{~nm}$, the photon-detection efficiency goes below $7 \%$ and falls to a value of about $2 \%$ at the very blue end of the spectrum.

The MR mode efficiency is specified to be identical to that of the HR mode, and indeed the two modes provide compatible results within measurement uncertainty. Figure 14 shows the efficiency as a function of wavelength and "as-delivered" seeing. The same considerations as for the HR mode apply, with the only difference being that much fewer observations are available at the time of writing and none could be obtained for nominal (photometric) conditions.

Figure 15 shows the eventual efficiency of the UHR mode as a function of wavelength and seeing. Considering the fiber diameter of $0.5^{\prime \prime}$, the obtained photon-detection efficiency exceeds expectations. 

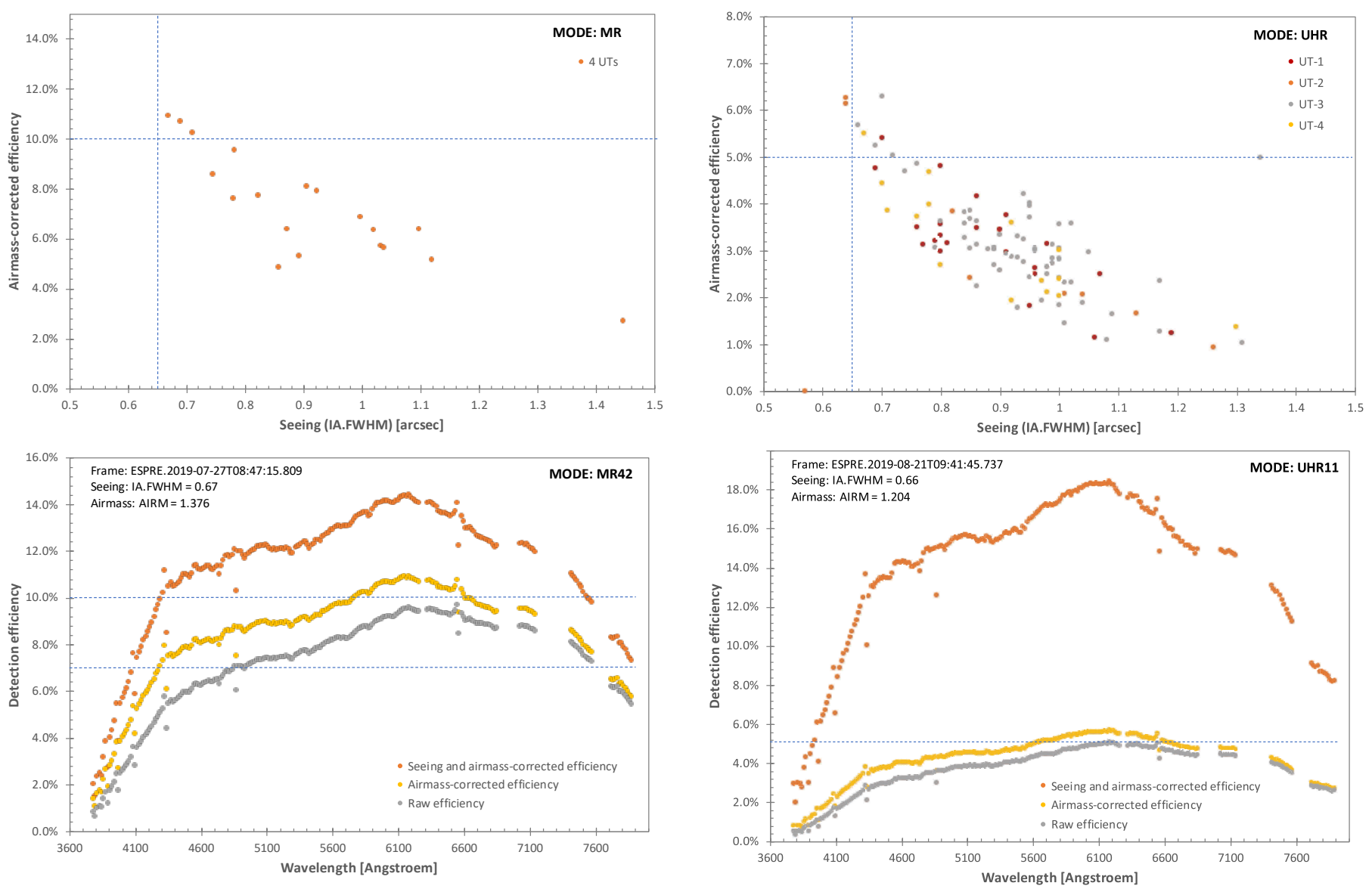

Fig. 14. Bottom: airmass-corrected efficiency (no atmospheric absorption) for the MR mode as a function of seeing (image quality IA.FWHM). Top: total photon-detection efficiency for the MR mode in binning $4 \times 2$ as a function of wavelength. An example of an image quality of IA.FWHM of $0.67^{\prime \prime}$ and airmass of 1.376 is shown.

The plots of the efficiency versus the seeing for the HR and the UHR modes show measurements obtained with all the UTs of the VLT. The fact that no systematic efficiency difference is observed between the telescopes demonstrates that the requirement of identical detection efficiency for each telescope is met. Furthermore, the fact that the MR-mode efficiency is very close to the HR-mode efficiency demonstrates that all four "channels" of the MR mode (4-UT configuration) have similar - and thus necessarily nominal - efficiency. Moreover, we observed with one UT using the MR fiber link and cycling though all four UTs individually, obtaining consistent figures for the throughput.

\subsubsection{Signal-to-noise ratio and precision}

Internal RV precision, that is to say, the fundamental precision that can be obtained if only photon noise limits the measurement, is estimated using the algorithms described in Bouchy et al. (2001). It should be recalled that the fundamental RV precision not only depends on the number of photons and the $\mathrm{S} / \mathrm{N}$ of the measurement, but also on the number and effective width of the spectral lines that are used to measure the RV. For this reason, the RV precision depends, on the one hand, on the resolving power of the spectrograph, and, on the other hand, on the spectral type and the rotational velocity of the star (among other broadening) if a stellar spectrum is observed. Consequently, the different observing configurations and instrument modes of ESPRESSO

Fig. 15. Bottom: airmass-corrected efficiency (no atmospheric absorption) for the UHR mode as a function of seeing (image quality IA.FWHM). The plot combines the measurements from all telescopes. Top: total photon-detection efficiency for the UHR mode in binning $1 \times 1$ as a function of wavelength. An example of an image quality of IA.FWHM of $0.66^{\prime \prime}$ and airmass of 1.204 is shown.

will not deliver identical fundamental precision under identical observational conditions. In the following, we will only discuss the HR mode in more detail since it is the standard mode designed for precise RV measurements.

In order to estimate the $\mathrm{S} / \mathrm{N}$ and the $\mathrm{RV}$ precision as well as to provide benchmarks for the standard HR11 mode, we analyzed GTO RV data from July to December 2019 (after the fiber upgrade). Figure 16 shows the $\mathrm{S} / \mathrm{N}$ obtained at a $550 \mathrm{~nm}$ wavelength in an extracted-pixel bin of $9 \mathrm{~m} \AA\left(0.49 \mathrm{~km} \mathrm{~s}^{-1}\right.$, no binning in spectral direction) as a function of target magnitude after they are normalized to the exposure time of $20 \mathrm{~min}$. Taking the upper envelope shown by the dashed line, we measure an $\mathrm{S} / \mathrm{N}$ on the order of $S / N=430$ for $V=8 \mathrm{mag}$ and $S / N=70$ for $V=12$ mag. Starting at $V=14 \mathrm{mag}$, a significant departure from the photon-noise limited behavior is observed.

We consider thus $V=14$ mag to be the "limiting magnitude" (transition between photon-noise and detector RON limit) in the HR11 mode. While the S/N obtained in the HR21 and HR42 binning modes is identical up to this limiting magnitude, the limiting magnitudes themselves are shifted to about $V \sim 16$ mag and $V \sim 18 \mathrm{mag}$, respectively. While the resolving power in the HR42 mode is reduced by about 5\% compared to the HR 21 or HR11 modes, this difference is barely noted, since stellar absorption lines are resolved to some extent. Figure 17 shows a comparison of a small portion of the spectrum of HD 85512 observed 


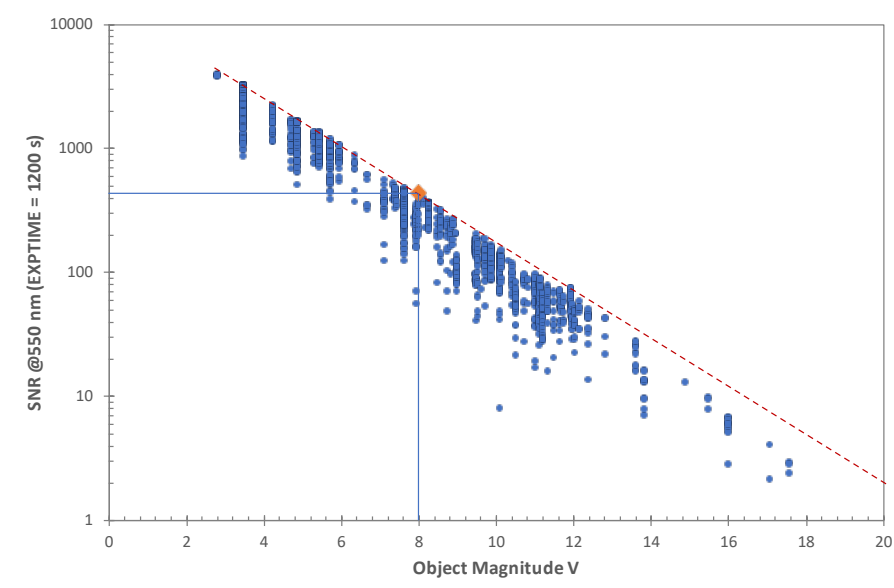

Fig. 16. Measured $\mathrm{S} / \mathrm{N}$ as a function of target magnitude for the HR11 mode (exposure time normalized to $1200 \mathrm{~s}$ ). The upper envelope given by the dashed line gives an indication of the $\mathrm{S} / \mathrm{N}$ reached under optimum astroclimatic conditions.

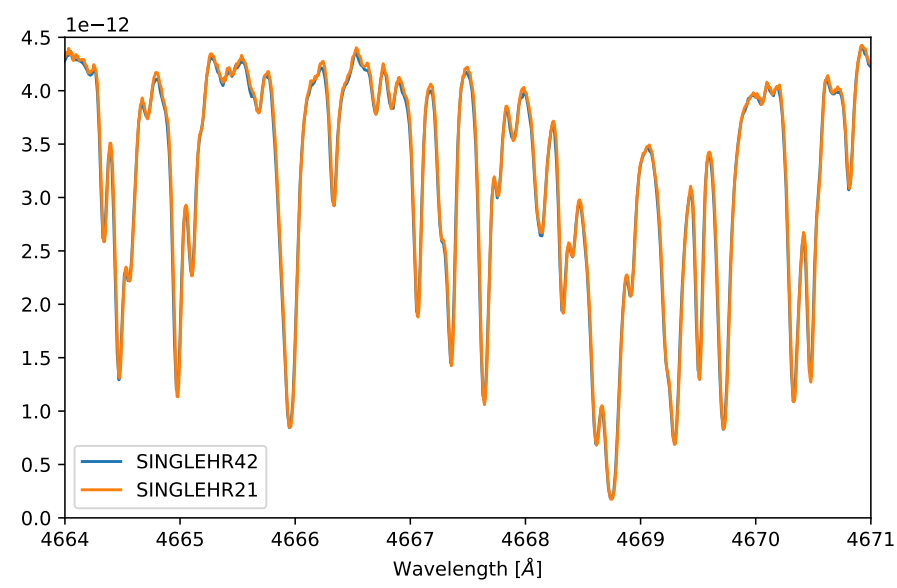

Fig. 17. Comparison of the spectra of HD 85512 obtained in the HR42 and HR21 modes.

with ESPRESSO in the HR21 and HR42 modes. The two spectra match almost perfectly after re-scaling, demonstrating the quality of the spectral extraction in the new HR42 mode and the negligible difference in spectral performance of the two modes. Furthermore, the spectral energy distribution (SED), or the $\mathrm{S} / \mathrm{N}$ measured as a function of wavelength, perfectly matches the one predicted by the official ETC (Fig. 18).

\subsection{Signal-to-noise performance at faint magnitudes}

On the night of July 7, 2019 (i.e., after the fiber upgrade), we observed the QSOJ16585649-5240283 of $V=17.07 \mathrm{mag}$ in HR21 mode with an integration time of $1800 \mathrm{~s}$. The image quality measured at the Nasmyth platform of the telescopes was of IA.FWHM $=1.1^{\prime \prime}$, while the DIMM seeing monitor indicated a seeing on the order of $0.9^{\prime \prime}$ during the exposure. The airmass was $A M=1.15$ on average. The average $\mathrm{S} / \mathrm{N}$ measured at $550 \mathrm{~nm}$ yields an $S / N=6.6$ in a spectral bin (extracted pixel) of $9 \mathrm{~m} \AA$ $\left(0.49 \mathrm{~km} \mathrm{~s}^{-1}\right)$, and thus perfectly in line with the extrapolated envelope shown in Fig. 16. In order to compare it with the technical requirements for the 1-UT configuration, this value has to be converted to an exposure of $t_{\exp }=3600 \mathrm{~s}$ and a spectral bin of $24 \mathrm{~m} \AA$, where it yields an $S / N=15$, well above the specified value of $S / N=10$.

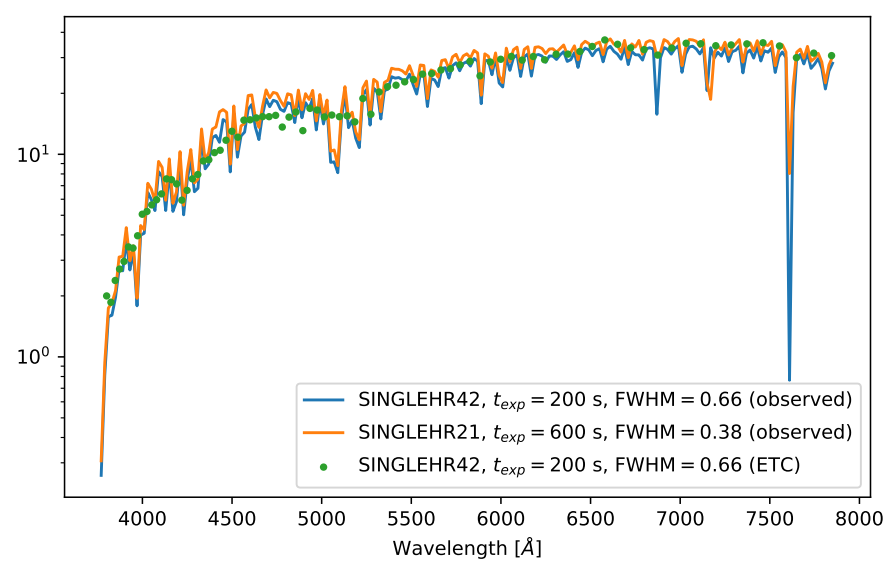

Fig. 18. Comparison of the S/N for HD 85512 as a function of wavelength as computed by the ETC with the values measured in the HR42 and the HR21 modes.

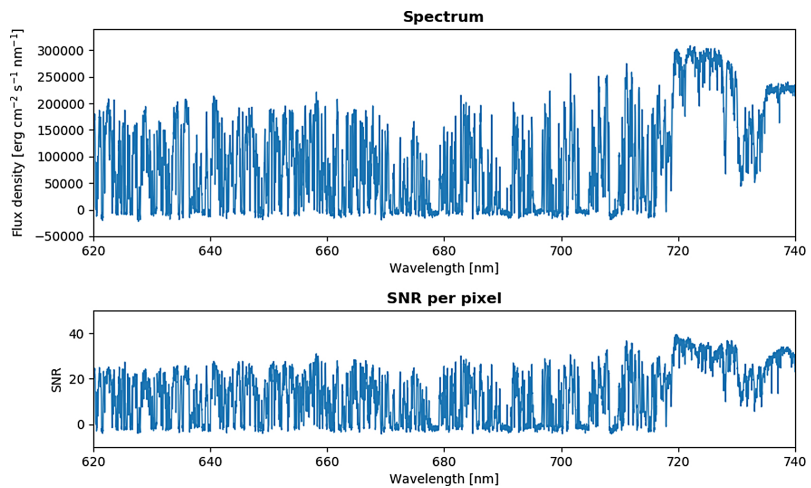

Fig. 19. Reduced spectrum of the QSO J013539.27-212628.4 ( $z=4.94$, $R=19.55 \mathrm{mag}, \mathrm{Li}$ et al. 2018) showing the Lyman-alpha emission and a portion of the Lyman forest. Reduction was carried out at the telescope with the ESPRESSO DRS (see Sect. 3.3.2), and the spectra were resampled by the ESPRESSO DAS (see Sect. 3.3.2, Cupani et al. 2016, 2019) using a fixed log-wavelength grid with a spectral bin of $2 \mathrm{~km} \mathrm{~s}^{-1}$ (resampled to $10 \mathrm{~km} \mathrm{~s}^{-1}$ for displaying purposes).

On the night of December 1, 2018, we observed the QSO J013539.27-212628.4 of $V \sim 20 \mathrm{mag}$ with an integration time of $1 \mathrm{~h}$ in the MR mode with four UTs (Fig. 19). The asdelivered image quality at the Nasmyth platform of the telescopes was IA.FWHM $=0.63^{\prime \prime}$, while the seeing monitor indicated a remarkable DIMM seeing on the order of $0.35^{\prime \prime}$ The airmass was of $A M=1.13$ on average. Data reduction was carried out at the telescope with the ESPRESSO DRS (see Sect. 3.3.2) and resampled by the ESPRESSO DAS (see Sect. 3.3.2, Cupani et al. 2016, 2019). The average S/N measured at $550 \mathrm{~nm}$ in HR84 mode yields, after subtraction of the background, $S / N=8.5$ measured in the spectral bin specified for the 4-UT configuration of $48 \mathrm{~m} \AA\left(2.63 \mathrm{~km} \mathrm{~s}^{-1}\right)$; the extracted spectral bin (four physical pixels) is about $35.5 \mathrm{~m} \AA\left(1.94 \mathrm{~km} \mathrm{~s}^{-1}\right)$ and contains a measured $\mathrm{S} / \mathrm{N}$ of about 7.3. The $\mathrm{S} / \mathrm{N}$ is computed in each spectral bin as the ratio between the collected flux and its formal error, propagated through reduction and resampling.

The reported measurement was obtained before the fiber-link intervention, thanks to which a gain of up to $40 \%$ in throughput was achieved for the MR mode. We extrapolate from this result that an $S / N=9.7$ is achieved after the fiber upgrade, which is very close to the specified value of 10 (spectral bin of $48 \mathrm{~m} \AA$, seeing $=0.8^{\prime \prime}$ ). Finally, we would also like to point towards successful science verification (SV) observations performed in 


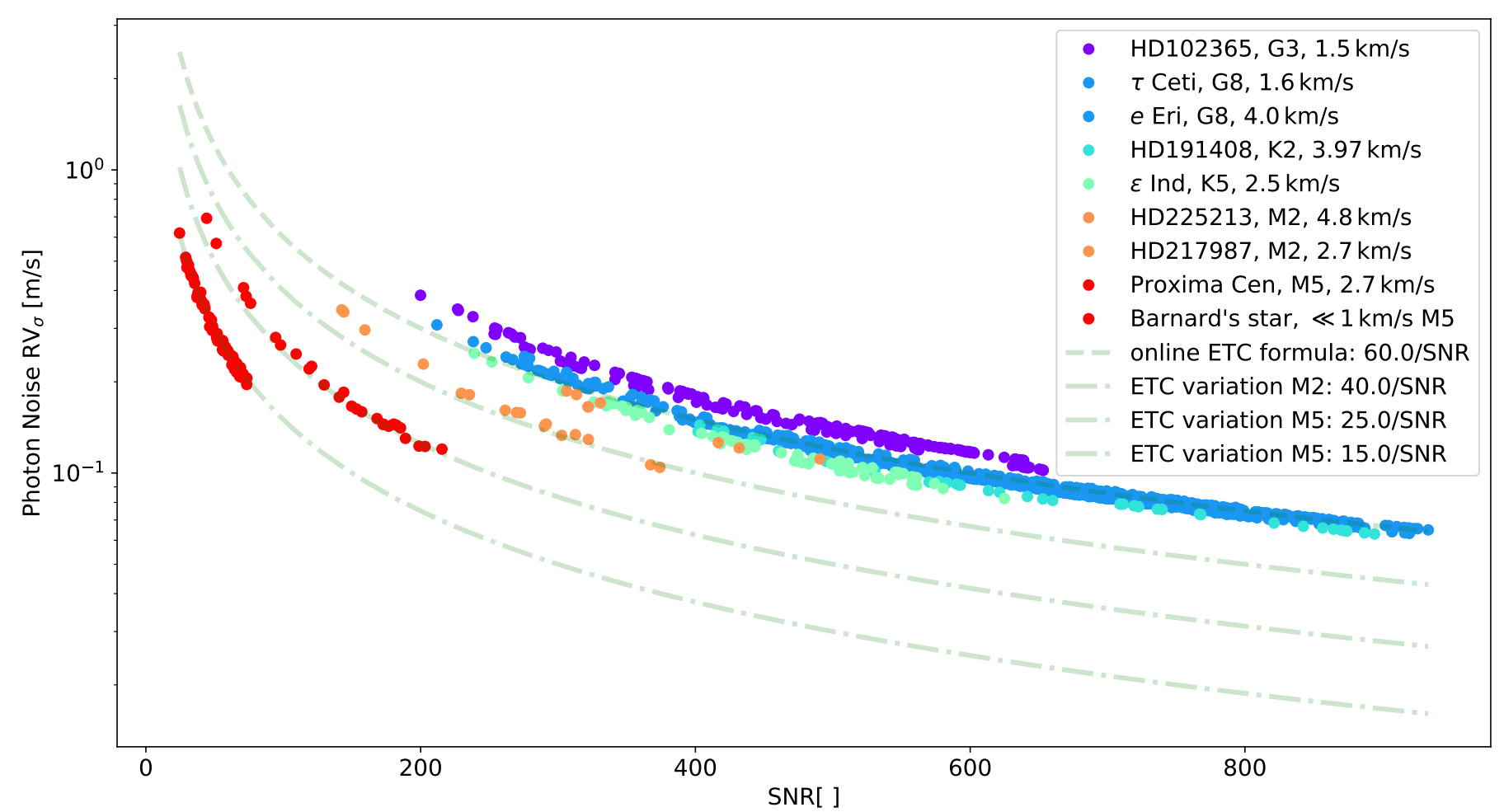

Fig. 20. Internal (photon-noise limited) RV precision as a function of $S / N$. The dots show the "on-spectra" determined RV error values for various standard RV targets for which the internal figure capture provides the spectral type and the $v \sin i$ value. The measured internal RV errors very nicely follow the scaled $1 /(\mathrm{S} / \mathrm{N})$ relation computed with the ETC and represented by the dash-dotted curves.

August 2019 on a very faint lensed galaxy (Vanzella et al. 2020) with $V \sim 21.5 \mathrm{mag}$ and $t_{\exp }=3600 \mathrm{~s}$ yielding to the significant detection of relevant emission lines despite the faint magnitude of the source and the high spectral resolution of the measurement.

\subsection{Radial-velocity performance}

In Fig. 20, we plot the measured on-sky internal (photon-noise limited) precision in the standard HR mode as a function of measured $\mathrm{S} / \mathrm{N}$ for several RV standards. At $S / N=430,10 \mathrm{~cm} \mathrm{~s}^{-1}$ precision is reached for $M$ stars, while for $K$ and $G$ dwarfs the RV precision lies between 10 and $20 \mathrm{~cm} \mathrm{~s}^{-1}$, depending on the rotational level of the star, thus slightly worse than the specified value.

In order to determine short- and medium-term RV repeatability, we observed, during the commissioning and in the frame of our GTO program, RV-standard stars taken from the HARPS high-precision program (Pepe et al. 2011). In Fig. 21, we show a time series recorded during commissioning 2A (March 1, 2018) on the $V=7.65$ mag K5-dwarf HD 85512, a nonrotating, very quiet star. The sequence covers about seven consecutive hours of observations in HR mode, binning $2 \times 1$, during which an average photon-noise error of $25 \mathrm{~cm} \mathrm{~s}^{-1}$ was obtained on each single exposure of $300 \mathrm{~s}$. The measured raw dispersion on the data is of $28 \mathrm{~cm} \mathrm{~s}^{-1} \mathrm{rms}$, very close to the photon-noise uncertainty. When the exposures are binned in groups of five data points, the dispersion is reduced to $8 \mathrm{~cm} \mathrm{~s}^{-1}$, again similar to the estimated average photon noise of $11 \mathrm{~cm} \mathrm{~s}^{-1}$.

The dependence of the measurement of the RV from the telescope was tested using observations of the same star. Although this star hosts a planet ( $K \sim 0.8 \mathrm{~m} \mathrm{~s}^{-1}, P \sim 58$ days), it is one of the quietest stars observable with ESPRESSO. Its magnitude is

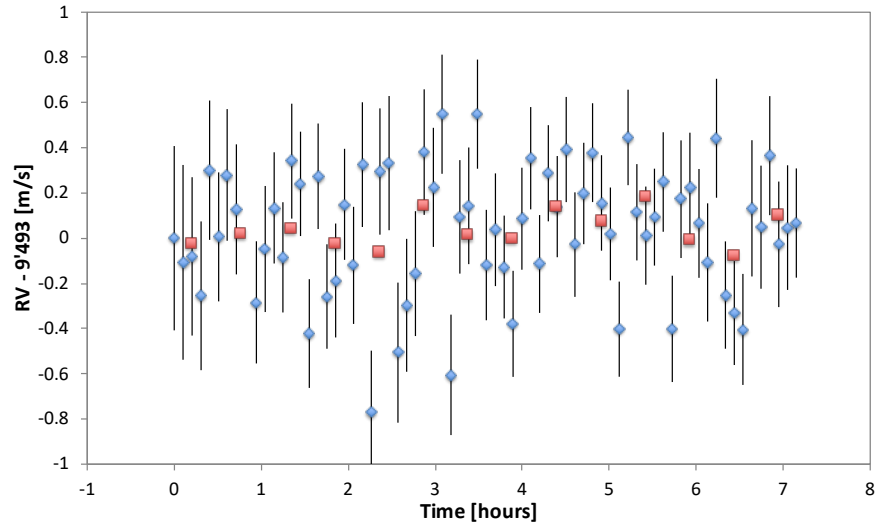

Fig. 21. RV series (blue diamonds) of $7 \mathrm{~h}$ on HD 85512. The red squares represent the binned data (five data points each).

such that we can achieve a photon noise smaller than $20 \mathrm{~cm} \mathrm{~s}^{-1}$ in 15 min of exposure. During a night with stable seeing and a clear sky, the star was observed in rapid sequence with all four UTs. The exposure time of $900 \mathrm{~s}$ allowed us to average the stellar acoustic pulsation ( $p$-modes). As the data points in Fig. 24 show, the RV variation between telescopes is within the $18 \mathrm{~cm} \mathrm{~s}^{-1}$ photon noise in this case. The RV variation observed when repeating the observation with UT1 and UT3 is attributed to stellar effects (Dumusque 2012).

In order to test the long-term "precision" (measurement repeatability), we collected measurement series of RV-standard stars observed during the first year of operations. We note that the series include the moment of the fiber upgrade in June 2019 and are likely to be affected by an instrumental RV offset. We advise ESPRESSO users to distinguish between ESPRESSO 


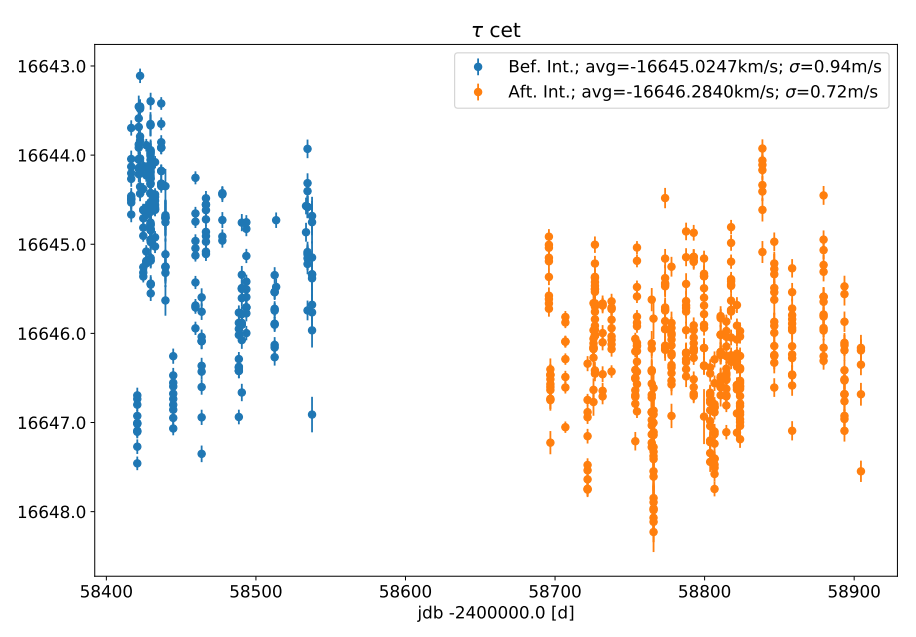

Fig. 22. Radial velocities obtained with ESPRESSO on $\tau$ Cet covering approximately one and half year of observations. Blue data points were taken before (and orange data points after) the fiber upgrade of June 2019.

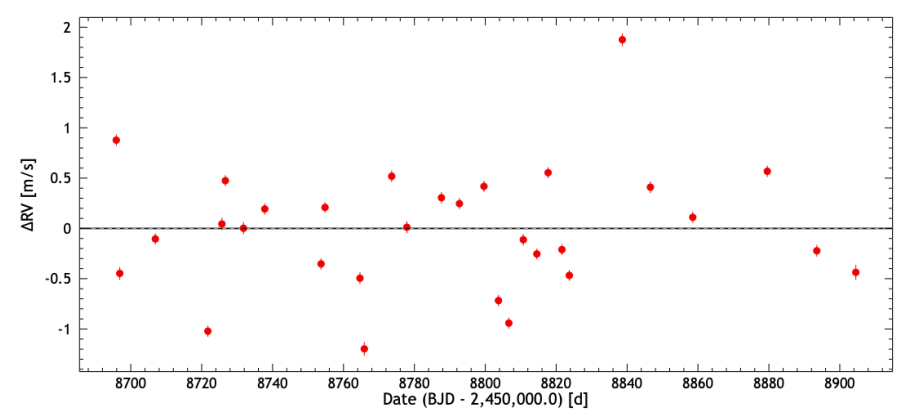

Fig. 23. Radial velocities obtained with ESPRESSO on $\tau$ Cet. Only data obtained after the fiber upgrade are shown. Each data point corresponds to a nightly bin. In this case, the dispersion is of $0.58 \mathrm{~m} \mathrm{~s}^{-1}$.

data taken before and after June 2019 by introducing a free offset parameter since it cannot be guaranteed that no instrumental RVoffset was introduced by this intervention on the spectrograph.

Figure 22 shows the radial velocities of HD 10700, one of the two stablest stars of the GTO Working-Group 1 program (blind search for rocky planets). Data points taken before the fiber upgrade are shown in blue, those after the intervention in orange. The raw scatter of the unbinned data obtained before the fiber upgrade is $0.94 \mathrm{~m} \mathrm{~s}^{-1}$, and the raw scatter after the fiber upgrade is $0.72 \mathrm{~m} \mathrm{~s}^{-1}$. We suspect that the higher dispersion in the early lifetime of the instrument is due to some "instabilities" introduced by operational issues (air-condition failures, thermal instabilities of the detector cryostat, etc.). The scatter of the radial velocities of $\tau$ Cet (HD 10700) decreases to $0.5 \mathrm{~m} \mathrm{~s}^{-1}$ when nightly bins are considered (Fig. 23). In summary, a long-term ( $\sim 1$ year) $\mathrm{RV}$ precision of better than $0.5 \mathrm{~m} \mathrm{~s}^{-1}$ is demonstrated, despite the intrinsic measurement limitations set by the astrophysical targets themselves. ESPRESSO delivers (non-photon-noise limited) RVs better than HARPS when compared over a similar time span, and the RV performance tested thus far is compatible with the $10 \mathrm{~cm} \mathrm{~s}^{-1}$-precision requirement.

The RV repeatability in the MR mode was analyzed on a one-hour timescale. It was found to be better than $50 \mathrm{~cm} \mathrm{~s}^{-1}$, consistent with the photon noise and significantly better than the $\mathrm{RV}$-precision requirement of $5 \mathrm{~m} \mathrm{~s}^{-1}$ for this mode. The test was repeated after the fiber-link exchange but with only three UTs
(UT1 was out of operation), obtaining a short-term repeatability of better than $40 \mathrm{~cm} \mathrm{~s}^{-1}$, consistent with photon noise.

The RV repeatability of the UHR mode was tested to better than $50 \mathrm{~cm} \mathrm{~s}^{-1}$ (13 $\mathrm{cm} \mathrm{s}^{-1}$ photon noise) in the short term (night). The measurement is dominated by the 5 min acoustic oscillations of the observed star (HD 10700, exposure time of $1 \mathrm{~min}$ ). There was no requirement specified for the RV-precision in the UHR mode, but we expect it to be comparable to the HR mode.

\subsection{Equivalence of Unit Telescopes}

The fact that ESPRESSO can operate with any of the four UTs calls for a very particular requirement, namely that any performance figure of ESPRESSO is independent from the telescope used. While transmission may vary between one telescope and another, for example due to the different ages of the mirror coatings (typically re-coated every two years), these differences are comparable to the absolute and chromatic variability introduced by the varying astroclimatic conditions (seeing, transparency and airmass, clouds). Despite these possible variations, we conducted on-sky tests to demonstrate that neither transmittance nor RVs measured with ESPRESSO depend on the UT on which the observations are carried out. Figure 24 shows RV measurements of the same star when switching from one telescope to another. Error bars show that the photonic RV uncertainty $\left(\sim 18 \mathrm{~cm} \mathrm{~s}^{-1}\right)$, and thus the telescope transmittance, remain essentially constant. Since RV precision depends linearly on $\mathrm{S} / \mathrm{N}$, this test also demonstrates that the throughput of ESPRESSO varies from one UT to another at a level below the effect induced by the variability of observing conditions. A similar statement holds true for the RV measurement: while cycling through the telescopes (first four measurements), no significant RV offset is observed. Returning to UT1 (fifth measurement), the same RV is obtained, within the error bar, as for the very first measurement. The second measurement on UT2 (sixth measurement) shows a 2-sigma deviation from the previous measurements. The RV dispersion of the series nevertheless remains compatible with the photonic error of $\left(\sim 18 \mathrm{~cm} \mathrm{~s}^{-1}\right)$. The test was repeated on other stars and under different conditions, and similar results were obtained. These results demonstrate that if RVoffsets are induced by changing the UT, they must be smaller than $20 \mathrm{~cm} \mathrm{~s}^{-1} \mathrm{rms}$.

Transmission and RV stability measurements are part of ESPRESSO's calibration plan and are monitored routinely. As for any of the ESO instruments, the "health" and performance of ESPRESSO are constantly recorded and published on dedicated web pages ${ }^{15}$.

\section{Guaranteed Time Observations with ESPRESSO}

In exchange for funding and building the ESPRESSO instrument, the ESPRESSO Consortium was awarded 273 nights of observations with the VLT (known as GTO), following a well-established and successful ESO scheme for funding second-generation instruments for the La Silla and Paranal observatories. Although formally granted to the principal investigator representing the Consortium, this person has to present observing proposals to ESO prior to the start of operations. The GTO proposals have to be approved by ESO's Observing Program Committee (OPC) before execution. All of the GTO proposals must follow the science program proposed by the

\footnotetext{
15 www. eso.org/observing/dfo/quality/ESPRESSO/common/ score_overview.html
} 


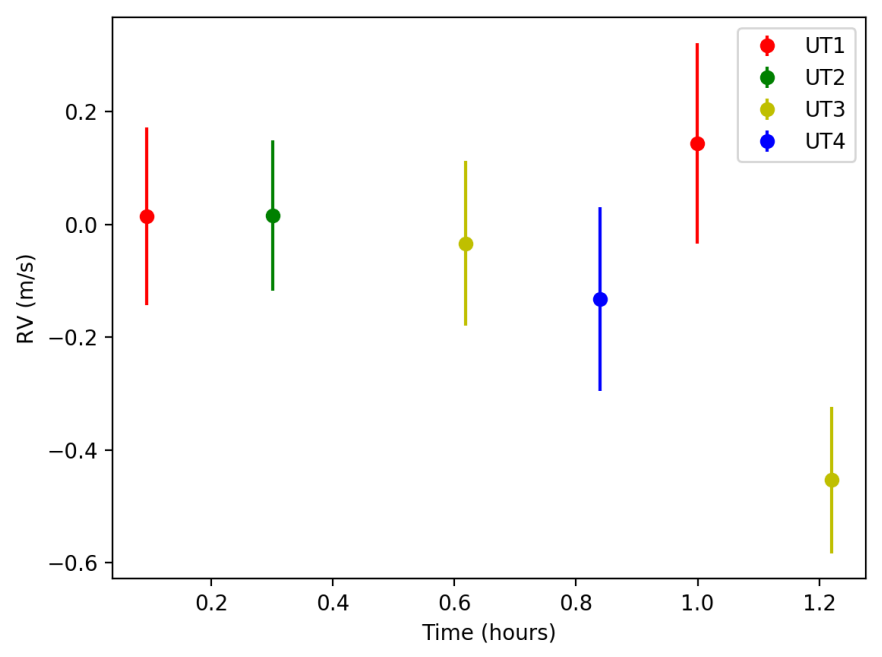

Fig. 24. RV series on the quiet star HD 85512 (K6V, $V=7.65 \mathrm{mag}$ ) obtained with ESPRESSO while cycling through the various UTs. Individual exposures of $600 \mathrm{~s}$ are shown. Error bars are essentially constant, demonstrating that the UTs have similar throughputs. The RV dispersion is compatible with the photon-noise induced RV error of about $18 \mathrm{~cm} \mathrm{~s}^{-1} \mathrm{rms}$ and with possible residual stellar $p$-modes that can be present even on late-type stars at this level of precision.

Consortium to ESO at the time of the signature of the construction agreement. In the case of ESPRESSO, the proposed program foresaw dedicating $80 \%$ of the granted GTO to the search for and characterization of exoplanets and $10 \%$ to the study of the (possible) variability of fundamental physical constants. The remaining $10 \%$ of the GTO had been reserved for "other science cases" of interest for the Consortium that would become topical at the time of the start of operations. In the following, we briefly describe the objectives, the contents, and the organization of the ESPRESSO Consortium's GTO program.

\subsection{Exoplanets}

The search for extrasolar planets is one of the fastest-expanding subjects in modern astrophysics. It was the detection of $51 \mathrm{Peg}$ b, the first extrasolar planet around a solar-type star, by Mayor \& Queloz (1995) that engaged a whole new community in the hunt for exoplanets. 51 Peg b was detected through the RV variation its orbit induced on its parent star, which became one of the most efficient techniques for the detection and characterization of exoplanets. After providing a significant wealth of information on hot-Jupiter characteristics, the RV technique has progressively revealed the existence of planets with lower masses down to the Earth and superEarth mass regime (including around our neighboring M-dwarf Proxima Cen (Anglada-Escudé et al. 2016). Over the past two decades, we have learned that at least $50 \%$ of stars in the solar neighborhood host a planet and that most of these can be found in multi-planetary systems (e.g., Lovis et al. 2011). Much of what is known about the exoplanet population comes from the detailed analysis of RV surveys (e.g., Udry et al. 2019). The efficiency of the RV technique is only challenged by that of space missions like Kepler (e.g., Borucki et al. 2011; Batalha et al. 2013), which have delivered the largest number of transit candidates for the more than 4000 planets known today.

The combination of transits and spectroscopy played a fundamental role in the detailed characterization of individual planets. Together, that is by the technique called "transit spectroscopy", they provide information on the parent star, precise measurement of planetary masses and radii, and, therefore, planetary densities, and allow the investigation of planetary atmospheres. High-fidelity spectroscopy and RVs are essential tools for achieving these goals. When officially proposed to ESO (before the first results provided by the Kepler mission and well before the discovery of Earth-mass planets around stars in the solar neighborhood), ESPRESSO had already been conceived to pursue the following scientific objectives in the exoplanetary field: the discovery and characterization of Earth-mass planets (possibly) within the HZs of their parent stars of spectral types GKM, the characterization of the atmospheres of individual exoplanets, and the precise mass determination of low-mass transiting planets discovered by the Kepler and Transiting Exoplanet Survey Satellite (TESS) missions. In what follows, we describe these three sub-programs.

\subsubsection{Blind search for rocky planets in the habitable zone}

One of the main science drivers for ESPRESSO is the detection of Earth-mass planets in the HZ around GKM stars. It was this driver that set the most stringent constraints on spectral resolution, thermo-mechanical stability, and illumination stability, ultimately aiming for $10 \mathrm{~cm} \mathrm{~s}^{-1}$ of RV precision. This enables the search for Earth-mass planets inside the HZs of solar-type stars, provided that these stars are "quiet" and sufficiently bright, thus pointing towards late- $\mathrm{G}, \mathrm{K}$ and $\mathrm{M}$ dwarfs of the solar neighborhood. Their proximity make them particularly suitable for follow-up studies regarding, for example, direct detection and characterization of their atmospheres. Starting in 2010, we performed several preparatory studies to define our target sample. We investigated the possible contamination by faint, spatially unresolved companions (Cunha et al. 2013) and the impact of micro-tellurics on precise RVs (Cunha et al. 2014), among other topics. To avoid being biased by previous RV surveys, we built our WG-1 catalog from scratch, performing a spectroscopic characterization for the stars that had not yet been observed at high spectral resolution. We obtained observing time on HARPS, HARPS-N, and UVES to complement HARPS observations retrieved from the ESO archive. For all candidates, we performed a detailed spectroscopic characterization as well as calculated the rotational velocity and chromospheric activity indices, among several other studies. The final target list contained 77 close-by, quiet, nonrotating stars covering a wide range of right ascension values and being thus observable the entire year. For these stars, the photon noise attained in a $15 \mathrm{~min}$ integration time (or $30 \mathrm{~min}$ for the faintest targets) allows us to detect the RV signal of a one Earth-mass planet orbiting inside the HZ. The details regarding target selection, spectral characterization, and further studies, along with reduced data access, are presented in Hojjatpanah et al. (2019).

During the first year and a half of observations, we narrowed down our sample to $\sim 45$ promising targets, of which 25 will be followed intensively and on which we expect to conduct an average of 60-70 visits by the end of the GTO. The remaining 30 targets were discarded due to the presence of stellar companions, or simply to avoid competition with other programs already following them.

It is worth mentioning that for several stars of this subprogram, the RV scatter is less than $1 \mathrm{~m} \mathrm{~s}^{-1}$; we stress that this value is computed without any data post-processing and with the RVs provided by the publicly available pipeline. For $\tau$ Cet, the $\mathrm{RV}$ scatter of the nightly binned RVs is of $50 \mathrm{~cm} \mathrm{~s}^{-1}$, as reported in a previous section. In Suárez Mascareño et al. (2020), the first 
paper of this sub-program, 63 ESPRESSO RV-points are used to independently confirm the planet Proxima Cen b, characterize the stellar activity signal on the star, and precisely measure the planetary signal. Once the planetary and stellar activity models are removed, the residuals scatter is at the level set by photon-noise of $\sigma_{\mathrm{RV}}=30 \mathrm{~cm} \mathrm{~s}^{-1}$, demonstrating unprecedented precision.

\subsubsection{Characterization of planetary atmospheres}

The study of planetary atmospheres from the ground (see e.g., Snellen et al. 2008; Redfield et al. 2008; Charbonneau et al. 2002) represents a breakthrough in our understanding of what exoplanets are made of (chemical composition), which is essential for determining planetary evolution and may be key to better understanding planet formation processes, for example, whether a planet was born in the orbit it is observed in or whether it has migrated over a large distance. This is the case of warm and hot exoplanets with short orbital periods that lie very close to their parent stars. These exoplanets are a natural laboratory for chemistry and, quite likely, formation studies. Their high atmospheric temperatures prevent condensation and the sinking of refractory species, thus making the atmospheric composition more representative of the whole chemical content. Before ESPRESSO, various atomic and molecular species were detected at optical wavelengths in Jupiter-mass planets with expected equilibrium temperatures typically above $\sim 1000 \mathrm{~K}$ (sodium, potassium, calcium, magnesium, neutral and ionized iron and titanium, titanium oxide, and hydrogen; see, for example, Hoeijmakers et al. 2019; Casasayas-Barris et al. 2019, and references therein). Water vapor, helium, and possibly methane and carbon-bearing species are also seen in hot massive exoplanets in the near-infrared (for example, see Nortmann et al. 2018; Sotzen et al. 2020, and references therein). Some warm Neptunes (e.g., GJ 436b and GJ 3470b) and the super-Earth GJ 1214 b, on the contrary, show cloudy atmospheres, which do not allow the determination of their composition. The least dense planets at small orbital distances from their host stars present atmospheres undergoing evaporation processes. These pioneering results have been possible through transit spectroscopy with the Hubble Space Telescope (HST) and Spitzer as well as ground-based facilities, including a pathfinder ESO program that uses HARPS and HARPS-N (Wyttenbach et al. 2017). Complementing these studies, high-resolution spectroscopy with HARPS has also been shown to be a promising method for detecting exoplanet atmospheres in reflection (Martins et al. 2015a). There seems to be a great variety of planetary atmospheres, and even though studies of individual outstanding exoplanets represent a highlight per se, large number of observations are needed to address relevant questions on planet diversity, formation, and evolution. The ESPRESSO GTO program will provide optical spectra of more than 30 exoplanetary atmospheres (from hot to warm planets) over the GTO duration and will contribute to the direct measurement of their atmospheric composition and structure.

One of the first ESPRESSO-GTO results is first the detection of an asymmetric absorption signature due to neutral iron during the primary transit of the ultra-hot giant planet WASP76b (Ehrenreich et al. 2020). This feature is blueshifted in the trailing limb (probably owing to the combination of planetary rotation and atmospheric wind), but no signature appears in the leading limb. We interpreted this as evidence of the condensation of gaseous iron, which is injected from the hot (day) side atmosphere onto the cool (night) side of the planet. In coming papers, the ESPRESSO-GTO team will show that this scenario likely happens in more planets with similar temperatures as those of WASP-76b and will report robust detections of atomic elements in the atmospheres of warm planets, such as lithium, that were not solidly established before (Tabernero et al., in prep.; Borsa et al., in prep.).

\subsubsection{RV follow-up of $\mathrm{K} 2$ and TESS transiting planets}

The Kepler space mission has revolutionized our knowledge of exoplanets by discovering thousands of small transiting planets with radii below $4 R_{\oplus}$. It has thereby confirmed that systems of short-period super-Earths and Neptunes are ubiquitous in our Galaxy; in fact, they appear to be a very frequent outcome of planet formation. However, the picture that emerged from the Kepler results has remained largely incomplete: The faintness of the host stars has been hampering a comprehensive characterization of the Kepler systems through ground-based follow-up. In particular, host star fundamental parameters such as stellar radii are only poorly known in many cases, leading to inaccurate planetary radii (Fulton et al. 2017; Van Eylen et al. 2018). Furthermore, planet masses could only be reliably measured in a limited number of systems that have sufficiently bright host stars (e.g., Buchhave et al. 2016). This situation improved with the advent of the $\mathrm{K} 2$ mission, which scanned the ecliptic plane in search of new transiting planets orbiting typically brighter stars than those of the original Kepler discoveries. Since 2018, another space mission has been further revolutionizing the exoplanet field: the TESS. TESS is currently performing an all-sky transit search from space and has already discovered hundreds of new transiting planets orbiting stars brighter than those discovered by $\mathrm{K} 2$. Intense follow-up efforts using HARPS-like instruments to characterize the super-Earths and Neptunes found by these missions have been ongoing for almost a decade, however still with a limited scope; only the more massive planets on shorter-period orbits are amenable to robust mass measurements.

ESPRESSO represents a breakthrough in this respect: Its 2 mag gain and improved RV precision with respect to HARPSlike instruments will, for the first time, enable a thorough exploration of the rocky planet population detected by K2 and TESS. These rocky worlds have radii below $\sim 1.8 R_{\oplus}$ and their expected masses are typically below 6-8 $M_{\oplus}$, although their mass distributions currently remain largely unconstrained. ESPRESSO observations will be able to constrain the internal composition of these objects, namely their iron/rock/water mass fraction, under the reasonable assumption that they do not have any $\mathrm{H} / \mathrm{He}$ envelopes. Moreover, ESPRESSO will explore how the properties of this population vary with stellar irradiation, stellar mass, planetary architecture, and even stellar composition (Santos et al. 2017), shedding new light on the formation and evolution pathways of these systems. Ultimately, ESPRESSO will be able to characterize transiting rocky planets within the $\mathrm{HZ}$ of their host star with unprecedented detail.

The ESPRESSO-GTO K2/TESS planet sample has been defined based on the following criteria: (a) confirmed or validated planet candidate; (b) planet radius $<2.0 R_{\oplus}$; (c) host star $V<14.5$ mag (spectral-type dependent); and (d) no precise mass measurement available. In addition, we intend to probe the rocky-to-gaseous transition at a given stellar irradiation level. This will allow us to constrain the evaporation processes that transform sub-Neptunes into naked rocky cores under the influence of extreme UV (XUV) irradiation from the host star. According to the results of Fulton et al. (2017), a particularly 
interesting irradiation range to carry out this experiment is 250-200× Earth's irradiation levels, where both gas-rich subNeptunes and gas-poor rocky planets appear to coexist. Mass and density measurements from ESPRESSO will thus probe the mass and density thresholds, leading to significant evaporation of planetary envelopes. The target sample for this experiment is the same as the one defined above, complemented in the following ways: (1) adding the larger planets that are in the same systems as the rocky planets defined above, and (2) adding a few more sub-Neptunes and Neptunes $\left(<4 R_{\oplus}\right)$ in other systems, which are under irradiation levels of 50-200× Earth's level. The main objective of the program is to measure precise masses and bulk densities for all these objects. In total, we aim at characterizing 50-100 small planets by the end of the GTO period.

To illustrate the potential of ESPRESSO and this subprogram, we refer to the case of the planetary system $\pi$ Men. $\pi$ Men is a bright, "naked-eye" G0V-star ( $V=5.6 \mathrm{mag})$ known to host a sub-stellar companion on a long-period and very eccentric orbit of $P_{\mathrm{b}} \sim 2000$ days. On September 6, 2018, NASA announced the discovery by TESS (Ricker et al. 2015) of a second planet, the transiting super-Earth $\pi$ Men c $\left(P_{\mathrm{c}}=6.27\right.$ days; $\left.R_{\mathrm{c}}=2 R_{\oplus}\right)$. Following the announcement, Huang et al. (2018) and Gandolfi et al. (2018) independently detected the spectroscopic orbit of $\pi$ Men c by analyzing archival RVs of HARPS and UCLES, and confirmed its planetary nature.

The brightness of the star makes $\pi$ Men a perfect target for testing the performance of ESPRESSO in measuring the masses and bulk densities of low-mass planets. From September 5, 2018, to March 25, 2019, we recorded therefore a total of 275 spectra and covered a time span of 201 days. The spectra were acquired with a typical exposure time of $120 \mathrm{~s}$, providing median $S / N=243$ per extracted pixel at $\lambda=500 \mathrm{~nm}$ and a median RV (internal) precision of $25 \mathrm{~cm} \mathrm{~s}^{-1}$. During each observing night, we collected series of multiple spectra at a rate of two to 12 consecutive exposures, leading to a typical in-night RV precision of the binned data points of about $10 \mathrm{~cm} \mathrm{~s}^{-1}$. Furthermore, TESS reobserved the host star during cycle 1 (sectors 4,8 , and 11-13) from October 2018 to July 2019, collecting 19 additional transits of planet c in short-cadence mode.

A detailed and complete analysis of the system architecture of $\pi$ Men is presented in a parallel paper by Damasso et al. (this same edition of the journal). The authors combined the exquisite RVs of ESPRESSO with the TESS photometry and with the Gaia and HIPPARCos astrometry. Besides refining the mass of the transiting super-Earth and reducing the error bars on its orbital parameters, the authors also constrained the mutual orbital inclination of the two planets, the real mass of the longperiod planet $b$, and the system architecture in general.

\subsection{Varying fundamental constants}

The standard model of particle physics depends on many $(\sim 27)$ independent numerical parameters that determine, inter alia, the strengths of the different forces and the relative masses of all known fundamental particles. There is no theoretical explanation for their actual value, but they nevertheless determine the properties of atoms, cells, stars, and the whole Universe. They are commonly referred to as the fundamental constants of nature, and our only definition for them is: any parameter that cannot be calculated from first principles in a given theoretical framework and therefore must be determined by experiment. Nevertheless, most of the modern extensions of the standard model predict a variation of these constants at some level (Uzan 2011; Bonifacio et al. 2014; Martins 2017).
For instance, in any theory involving more than four spacetime dimensions, the constants we observe are merely 4D shadows of the truly fundamental high-dimensional constants. The 4D constants will then be seen to vary as the extra dimensions change slowly in size during their cosmological evolution. The most common examples in modern cosmology are the quintessence models for dark energy, in which the underlying dynamical scalar field is naturally expected to couple with the electromagnetic sector of the theory and lead to a variation of the fine-structure constant (Carroll 1998); thus tests of the stability of the fine-structure constant are a competitive cosmological probe (Martins et al. 2015b). Earth-based laboratories can measure the local drift rate of these constants. For the fine structure constant $\alpha$, this has been constrained to a few parts per $10^{-17} \mathrm{yr}^{-1}$ (Rosenband et al. 2008). This is more than six orders of magnitude slower that the typical cosmological drift rate (viz. $10^{-10} \mathrm{yr}^{-1}$ ), showing that any such scalar field must be evolving very slowly.

Astronomical observations have great potential for probing the stability of fundamental constants at very large distances and in the early Universe, and therefore identifying any slow but nontrivial evolution. In fact, the transition frequencies of the narrow metal absorption lines observed in the spectra of distant quasars are sensitive to $\alpha$, as first pointed out by Bahcall $\&$ Schmidt (1967), and those of the rare molecular hydrogen clouds are sensitive to $\mu$, the proton-to-electron mass ratio, as first pointed out by Thompson (1975).

With the advent of $10 \mathrm{~m}$ class telescopes, observations of spectral lines in distant QSOs provided the first hints that the value of the fine structure constant might change over time, being lower in the past by about $6 \mathrm{ppm}$ (Webb et al. 1999; Murphy et al. 2004). The addition of another 143 VLT/UVES absorbers have revealed $4 \sigma$ evidence for a dipole-like variation in $\alpha$ across the sky at the $10 \mathrm{ppm}$ level (Webb et al. 2011; King et al. 2012). Several other constraints from higherquality spectra of individual absorbers exist (Molaro et al. 2013; Evans et al. 2014; Murphy et al. 2016; Kotus et al. 2017; Bainbridge \& Webb 2017; Murphy \& Cooksey 2017) but none of them directly support or strongly conflict with the $\alpha$ dipole evidence, and a possible systematic producing opposite values in the two hemispheres is not easy to identify.

In order to probe $\mu$, the $\mathrm{H}_{2}$ absorbers need to be at a redshift $z>2-2.5$ to place the Lyman and Werner $\mathrm{H}_{2}$ transitions redwards of the atmospheric cutoff. Only five systems have been studied so far, with no current indication of variability at the level of $10 \mathrm{ppm}$ (Rahmani et al. 2013). At lower redshifts, precise constraints on $\mu$-variation are available from the radioand millimeter-wave spectra of cool clouds containing complex molecules such as ammonia and methanol (Levshakov et al. 2013). Other techniques involving radio spectra typically constrain combinations of constants by comparing different types of transitions (e.g., electronic, hyperfine, rotational, etc.).

Extraordinary claims requ ire extraordinary evidence, and a confirmation of variability with high statistical significance - in practice, at the level of $1 \mathrm{ppm}$ - is of crucial importance. Only a high-resolution spectrograph that combines a large collecting area with extreme wavelength precision can provide definitive clarification. A relative variation in $\alpha$ or $\mu$ of $1 \mathrm{ppm}$ leads to velocity shifts of about $20 \mathrm{~m} \mathrm{~s}^{-1}$ between typical combinations of transitions. ESPRESSO is therefore ideal for this task. About $10 \%$ of the GTO time will be used to collect spectra of a sample of carefully selected, relatively bright and well studied QSOs to significantly improve current measurements of $\alpha$ as well as $\mu$ with the aim to break the $1 \mathrm{ppm}$ precision. 
The proposed observations, a first version of which is summarized in Leite et al. (2016), will test the universality of physical laws in an unexplored regime, which directly impacts fundamental physics and theoretical cosmology. While the impact of confirmed spacetime variations is self-evident, more stringent upper bounds on these variations are also important and will lead to improved constraints on the parameter space of various theoretical paradigms that predict their variability (Martins 2017; Alves et al. 2017; Martins \& Vacher 2019).

\section{3. "Other science cases"}

The remaining $10 \%$ of the GTO was not allocated to a specific science case but left available for ad hoc proposals that arise during the execution of the GTO program. Incoming ideas are discussed and reviewed internally within the ESPRESSO-GTO Science Team before every call for proposals for the following observing period at ESO. Several programs have been or are being executed this way and have led to results that will be partially presented in the next section.

\subsubsection{Are very metal-poor stars binaries?}

The most metal-poor stars in the Galaxy are the most ancient fossil records of chemical composition and can provide clues on pre-Galactic phases and the nature of the stars that synthesized the first metals. Masses and yields of Population III stars can be inferred from the observed elemental ratios in the most metal-poor stars (Heger \& Woosley 2010). One crucial question is how to explain the mere presence of very metal-poor lowmass stars. Indeed, for a long time, the first stars formed have been thought to be very massive, but the recent discovery of several very metal-poor stars with $[\mathrm{Fe} / \mathrm{H}] \leq-5.0$ have presented an entirely new picture (Caffau et al. 2011).

There are about a dozen stars known with $[\mathrm{Fe} / \mathrm{H}]<-4.5$ reaching down to $[\mathrm{Fe} / \mathrm{H}] \approx-7$, in other words, 10 million times below solar. All but two show high-C overabundance (CEMP stars). We are carrying out ESPRESSO observations in order to probe their binary nature by means of accurate RV measurements. The observations will allow us to understand if $\mathrm{C}$ comes from a companion asymptotic giant branch (AGB) star or if it is related to a different kind of object, such as Weak SNe or Spinstars; they will also provide unique information on the nature of the first stars in the Universe and on the first elemental enrichment.

The vast majority of known stars of ultra-low metallicity are known to be enhanced in carbon and to belong to the lowcarbon band $(\mathrm{A}(\mathrm{C}) \leq 7.6)$. It is generally, although not universally, accepted that this peculiar chemical composition reflects the chemical composition of the gas cloud out of which these stars were formed. The first ultra metal-poor star discovered, HE 0107-5240, is also enhanced in carbon and belongs to the low-carbon band. It has recently been claimed that its peculiar composition could be due to mass-transfer to a formerly AGB companion (Arentsen et al. 2019). ESPRESSO observations obtained in 2018 demonstrate unambiguously that the RV of HE 0107-5240 increased between 2001 and 2018 and that the star is indeed a binary (Bonifacio et al. 2020). However, binarity does not necessarily imply mass exchange, and the nature of the companion remains undetermined. The companion could be either a white dwarf or an unevolved star. The binarity of such an object holds important information on the formation of small mass objects from a cloud without elements able to cool the gas and facilitate the first condensations.

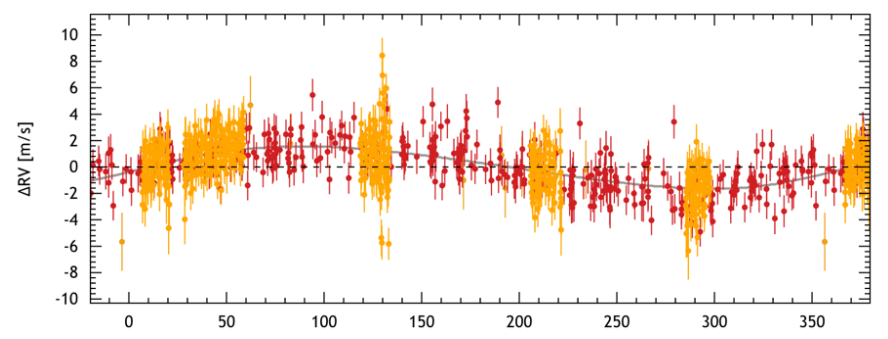

Fig. 25. ESPRESSO (yellow) and HARPS (red) radial velocities of HD 40307 phase-folded to the 4.31 day period of the inner super-Earth hosted by the star.

High-resolution simulations of star formation from primordial gas have shown the importance of the fragmentation processes (Greif et al. 2011). This provides a way of producing low-mass stars even in the absence of efficient cooling mechanisms, favoring the formation of binary systems.

\subsubsection{Asteroseismology of K-dwarf stars}

So far, no $p$-mode oscillations have been detected on solartype stars with spectral types later than K1V (e.g., $\alpha$ Cen B Carrier \& Bourban 2003; Kjeldsen et al. 2005). An estimate of the oscillation-mode amplitude, frequency domain, frequency of the maximum amplitude $v_{\max }$, and large splitting $\Delta v$ in a mid-K dwarf with an effective temperature $T_{\text {eff }}$ lower than $5000 \mathrm{~K}$ will allow us to refine the scaling relation from the Sun (Kjeldsen \& Bedding 2011) at the cool edge of the main sequence. The frequency at which a solar-like oscillator presents its strongest amplitude $v_{\max }$ scales with the surface gravity $\log g$ and effective temperature $T_{\text {eff }}$. The detection of $p$-mode oscillations in $\mathrm{K}$ dwarfs is still out of reach of photometric space missions, and the level of RV precision required is out of reach of any spectrograph other than ESPRESSO.

HD 40307 is one of the brightest mid-K dwarfs and is well located for a long time-series acquisition from the Southern Hemisphere. According to HARPS monitoring (Díaz et al. 2016), HD 40307 is a quiet star with $\log R_{h k}^{\prime}$ of -4.94 , which presents a magnetic cycle with a period of about 10 years and hosts a four-planet system. According to its effective temperature $(4977 \mathrm{~K})$, luminosity $\left(0.228 L_{\odot}\right)$, and mass $\left(0.77 M_{\odot}\right)$, HD 40307 is expected to have p-mode oscillations with an amplitude of $6-10 \mathrm{~cm} \mathrm{~s}^{-1}$ and a typical period of $2.5-3 \mathrm{~min}$ as well as a large separation of modes $\Delta v \sim 220-230 \mu \mathrm{Hz}$.

In December 2018, we conducted an asteroseismological campaign on HD 40307 with ESPRESSO over five nights. The detailed analysis of the RV time series and spectroscopic activity indicators (CCF FWHM, bisector span, $\mathrm{CaII}, \mathrm{H} \alpha$, etc.) will be presented in Bouchy et al. (in prep.). During this campaign, a total of 1150 measurements were obtained. The exposure time was set to $30 \mathrm{~s}$. The duty cycle was close to $78 \mathrm{~s}$ due to increased overhead time, which has since been slightly reduced. The average photon-noise uncertainty per exposure is $95 \mathrm{~cm} \mathrm{~s}^{-1}$. Figure 25 shows the ESPRESSO and HARPS radial velocities phase-folded to the 4.31 day planet of the HD 40307 system. ESPRESSO's exposure time was 30 times shorter than that of HARPS ( $900 \mathrm{~s})$. The typical dispersion of ESPRESSO RVs is $110 \mathrm{~cm} \mathrm{~s}^{-1}$ and not limited by the photon-noise. Figure 26 presents the RV dispersion as a function of the number of binned data points. One can see that by binning six data points ( $8 \mathrm{~min}$ bin), the dispersion is reduced to less than $50 \mathrm{~cm} \mathrm{~s}^{-1}$ and the additional noise (instrumental and stellar) is at the level of 


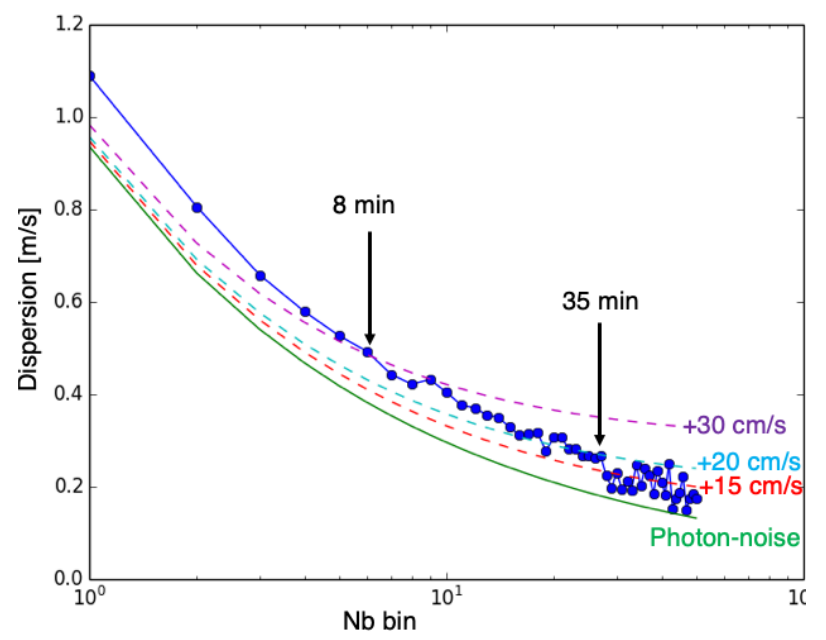

Fig. 26. Dispersion of the RV time series on HD 40307 as a function of the number of binned data points.

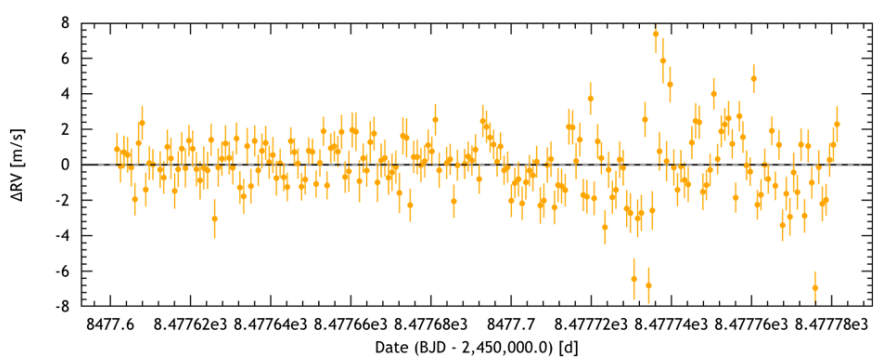

Fig. 27. ESPRESSO RV sequence on HD 40307 from December 24, 2018, showing evidence of $p$-mode excitation and damping.

$30 \mathrm{~cm} \mathrm{~s}^{-1}$. By averaging 27 data points (35 min bin), the dispersion is further reduced to below $20 \mathrm{~cm} \mathrm{~s}^{-1}$. These values suggest that the binning process progressively damps the signals that have a stellar origin. Hence, Fig. 26 leads us to believe that the residual instrumental noise must be at the level of only $10 \mathrm{~cm} \mathrm{~s}^{-1}$.

We find weak evidence of $p$-mode detection at a level of only $3-4 \mathrm{~cm} \mathrm{~s}^{-1}$, very close to our detection limit, slightly below the expected amplitude, and with a large separation of modes close to $220 \mu \mathrm{Hz}$. Furthermore, we expected to see the excitation and damping of $p$-mode oscillations on the timescale of a few hours. Figure 27 shows the RV time series obtained on December 24, 2018. The RV jitter increases suddenly after BJD $=8477.71$ and with a modulation of $2.5 \mathrm{~min}$. This pattern could not be linked to any (known) instrumental parameters, and we think that it shows the onset of coherent pulsation modes in realtime, a snapshot of a "stellar quake".

\subsubsection{An ESPRESSO measurement of primordial deuterium}

Observations of the abundances of the primordial elements produced in the Big Bang nucleosynthesis (BBN) provide one of the earliest (first few minutes) probes of the cosmological scenario, and any departure from the standard values and from homogeneity can be the smoking gun of new physics. Among the various elements and isotopes $\left(\mathrm{He}^{3}, \mathrm{He}^{4}, \mathrm{Li}\right)$, deuterium is known to be a powerful diagnostic, in particular providing the best constraint on the baryon abundance thanks to its sensitivity and monotonic dependence on the baryon-to-photon ratio, $\eta_{10}$.

For nearly 20 years, primordial deuterium abundance has been measured with the ratio of D I to H I column densities in absorption systems observed in high-redshift quasar spectra. This is not an easy task: high-resolution data have been used to this end, and both high $\mathrm{S} / \mathrm{N}$ and a strict control on systematics are required. Systematics can be related to the process of measurement (continuum fitting, H I Lyman- $\alpha$ line contamination, deblending of the velocity structure) as well as to external possible correlations of the abundance with the column (over-)density, metallicity, and/or redshift of the absorber.

The relative large scatter observed in the $\mathrm{D} / \mathrm{H}$ values throughout the era of accurate measurements has been typically ascribed to underestimates of the systematic errors. In a recent paper, Cooke et al. (2018) selected only seven absorbers that are able to provide a significant measurement. Their results turn out to be compatible with a uniform primordial $\mathrm{D} / \mathrm{H}=(2.53 \pm 0.03) \times 10^{-5}$ and consistent within $2 \sigma$ with the baryon density measurable from the Planck cosmic microwave background (CMB) observations.

ESPRESSO, with its characteristics of stability and wavelength accuracy, can provide measurements with much lower systematics. Furthermore, observations in 4-UT configuration allow us to observe a bright quasar with a resolution $\sim 1.5-1.75$ times larger than those normally obtained with UVES at VLT or HIRES at Keck. A larger resolution is critical for improving the determination of the continuum level and the characterization of the velocity structure of systems.

In August 2018, we observed the quasar PKS 1937-1009 for $5 \mathrm{~h}$ with ESPRESSO in 4-UT configuration. This is one of the brightest quasars $(V=16.95 \mathrm{mag})$ in the southern sky with a relatively high emission redshift $\left(z_{\mathrm{em}} \simeq 3.787\right)$, allowing us to observe its Lyman forest in the ESPRESSO wavelength range. In the spectrum of this object there are two Lyman limit systems $\left(\log \left(N_{\mathrm{HI}}\right) \sim 18\right)$ for which primordial deuterium measurements were carried out at $z \simeq 3.256$ and at $z \simeq 3.572$ (see Riemer-Sørensen et al. 2015, 2017 and references therein). The analysis of the ESPRESSO spectra (D'Odorico et al., in prep.) is improving our knowledge of the chemical abundances of the two systems, while the $\mathrm{S} / \mathrm{N}$ in the Lyman forest will probably need to be increased to obtain a more accurate determination of the $\mathrm{D} / \mathrm{H}$ abundances than previously reported.

\subsubsection{Small-scale structure of the intergalactic medium}

A number of studies on the large-scale properties of the intergalactic medium have concentrated on the crucial problem of the physical scale of Lyman absorbers. Observations (e.g., D'Odorico et al. 1998, 2002; Rauch et al. 2005) have shown that their typical size is large and that they must be part of the general large-scale structure. Ionization arguments, analytical and Monte Carlo modeling of absorption in double lines of sight (Findlay et al. 2018 and references therein), and cosmological hydro-simulations all suggest that the absorbing structures are part of a filamentary cosmic web that is expected to follow the Hubble flow on large (megaparsec) scales, that is to say, on scales that are at least larger than the typical coherence length of these structures. On intermediate scales (on the order of $100 \mathrm{kpc}$ ), the effects of gravitational collapse may become more pronounced, with the gas gaining kinetic energy in the galactic potential wells, while on the smallest (subkiloparsec) scales, processes like stellar winds and supernova explosions should be the dominant sources of momentum.

We have taken advantage of the unique capabilities of ESPRESSO to explore the kiloparsec and subkiloparsec scales and gain insight into the feedback processes governing the formation of galaxies as well as to verify whether systematic 


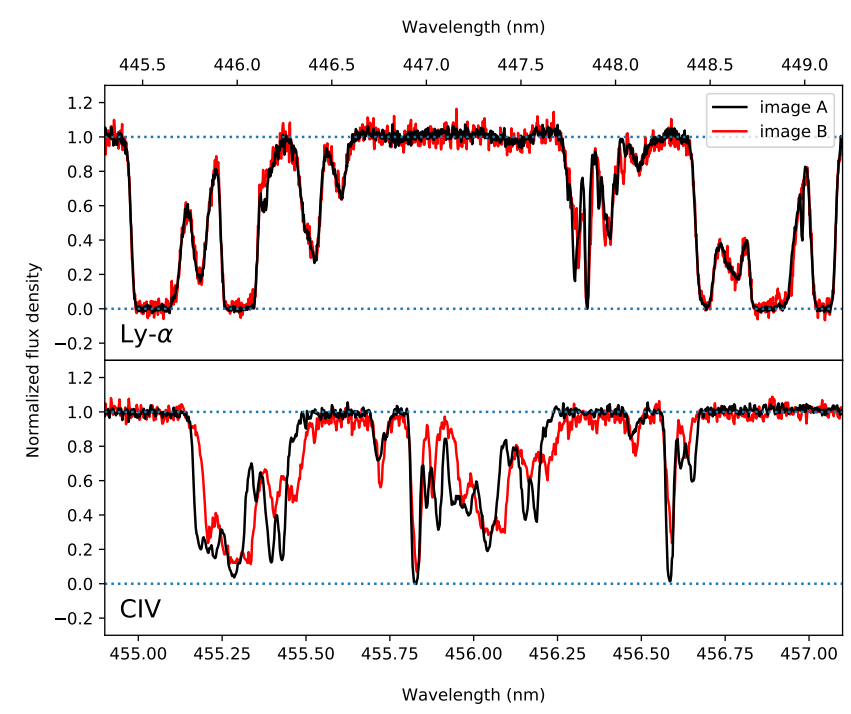

Fig. 28. Portion of the spectrum of Q0142-100AB observed with ESPRESSO. Top: region of Lyman-alpha forest (with a few metal interlopers), which shows a remarkable stability. Bottom: complex CIV $\lambda \lambda 1548,1550$ absorption system, which shows a different velocity structure along the two lines of sight (image A and B, black and red curves, respectively).

variations of the dynamical and ionization states in the Lyman forest might affect the Sandage test (Loeb 1998; Cristiani et al. 2007; Liske et al. 2008) in future experiments. We observed a bright lensed QSO with emission redshift 2.72 and image split on the sky of $2.5^{\prime \prime}$ with ESPRESSO in 4-UT configuration. The lines of sight of Q0142-100AB span the Ly-alpha redshift from $z=2.7$ down to 2.2 and represent, due to the geometry of the lens, transverse scales from about 15 physical kiloparsecs down to the subkiloparsec range. This is the brightest known lens in the southern sky for which the Lyman forest is observable by ESPRESSO and the separation of at least two images of the lens is above $2^{\prime \prime}$ (in order to avoid fiber flux contamination). The Lyman forest on these scales shows a remarkable stability: Pairs of absorptions observed in the two lines of sight display the same column density, Doppler parameter, and redshift within the measurement error of the photon statistics (Fig. 28, top). In particular, the velocity differences appear to be below the observational rms limit of $1.1 \mathrm{~km} \mathrm{~s}^{-1}$. On the contrary, metal lines, and the CIV species in particular (Fig. 28, bottom), show strong variations, indicating that they must have variations of structures on scales on the order of $10^{2} \mathrm{pc}$ (Cristiani et al., in prep.).

\section{Conclusions}

ESPRESSO at VLT offers the astronomical community highresolution, high-fidelity, full-range visible spectroscopy on a $10 \mathrm{~m}$ class telescope. The instrument's unique features open up a new parameter space in ground-based observations that can address a large variety of scientific cases. In this paper, we presented the ESPRESSO instrument and provided information on its characteristics as well as advice on how to carry out observations.

Using on-sky tests carried out during the commissioning phase and the first year of operations, we demonstrated that all the main performance requirements have been met. First, high resolving power of $R \sim 140000$ and $R>190000$ is provided in the 1-UT configuration over the full wavelength range from 378.2 to $788.7 \mathrm{~nm}$. In 4-UT configuration, a resolving power of $R \sim 70000$ is achieved despite having to cope with the etendue of four $8 \mathrm{~m}$ telescopes. Second, the overall throughput, from the top of the atmosphere to the detector, peaks at $10 \%$ in both the 1-UT and the 4-UT configurations. Third, short-term (night) RV measurement are limited by photon noise down to the $10 \mathrm{~cm} \mathrm{~s}^{-1}$ level. Over the long term (months), a repeatability of better than $50 \mathrm{~cm} \mathrm{~s}^{-1}$ rms has been demonstrated. Given the fact that photon noise and stellar jitter affect single RV measurements, this value provides an upper limit for the instrumental precision. Last, we have demonstrated that throughput and RV measurement do not depend on the used telescope. This fact allows for a unique versatility of the VLT in its operations.

We presented our GTO program that is being carried out on ESPRESSO. Due to the time-series nature of the RV observations, and in particular the blind-search subprogram, we were not expecting to produce results shortly after the start of operations. Two other subprograms, aiming at the RV followup of TESS and K2 and at observing planetary atmospheres through transit spectroscopy, however, immediately benefited from the unique spectroscopic capabilities of ESPRESSO (e.g., Ehrenreich et al. 2020). Furthermore, the simultaneous start of TESS and ESPRESSO operations enabled exceptional synergies between spaced-based transit searches and ground-based $\mathrm{RV}$ observations. The results on $\pi$ Men presented in Damasso et al. (this same edition of the journal) are the direct demonstration of the power of simultaneous high-precision photometric and spectroscopic observations.

Acknowledgements. ESPRESSO is a 25 MEUR project that lasted about 10 years and involved dozens of collaborators. The large number of co-authors is a manifestation of this effort, but it is not able to represent adequately the passion of all the people who made this project possible. It is for this simple reason that F.P. would like to express his profound gratitude to technical and administrative assistants, technicians, engineers, managers and science collaborators who helped navigating through this challenging adventure with flexibility, dedication and creativity. We cannot avoid problems in Life, but by working with Friends, all together, we can overcome them. F.P. and C.L. would like to acknowledge the Swiss National Science Foundation (SNSF) for supporting research with ESPRESSO through the SNSF grants nr. 140649, 152721, 166227 and 184618. The ESPRESSO Instrument Project was partially funded through SNSF's FLARE program for large infrastructures. This work has been carried out in part within the framework of the NCCR PlanetS supported by the Swiss National Science Foundation. M.D. acknowledges financial support from Progetto Premiale 2015 FRONTIERA (OB.FU. 1.05.06.11) funding scheme of the Italian Ministry of Education, University, and Research. We acknowledge the computing centers of INAF - Osservatorio Astronomico di Trieste/Osservatorio Astrofisico di Catania, under the coordination of the CHIPP project, for the availability of computing resources and support. This work was supported by FCT - Funda cão para a Ciência e Tecnologia - through national funds and by FEDER through COMPETE2020 - Programa Operacional Competitividade e Internacionaliza c ao - by these grants: UID/FIS/04434/2019; UIDB/04434/2020; UIDP/04434/2020; PTDC/FIS-AST/32113/2017 \& POCI-01-0145-FEDER-032113; PTDC/FIS-AST/28953/2017 \& POCI01-0145-FEDER-028953; PTDC/FIS-AST/28987/2017 \& POCI-01-0145FEDER-028987. V.A., S.C.C.B., O.D.S.D., J.P.F. and S.S. acknowledge support from FCT through work contracts $n^{\circ}$ s IF/00650/2015/CP1273, IF/01312/2014/CP1215/CT0004, DL 57/2016/CP1364/CT0004, DL 57/2016/CP1364/CT0005, IF/00028/2014/CP1215/CT0002. J.L.-B. has been funded by the Spanish State Research Agency (AEI) Projects No.ESP201787676-C5-1-R and No. MDM-2017-0737 Unidad de Excelencia "María de Maeztu" - Centro de Astrobiología (INTA-CSIC). V.B. acknowledges support by the Swiss National Science Foundation (SNSF) in the frame of the National Centre for Competence in Research "PlanetS". This project has received funding from the European Research Council (ERC) under the European Union's Horizon 2020 research and innovation program (project Four Aces, grant agreement No 724427). R.R., C.A.P., J.I.G.H. and A.S.M. acknowledge financial support from the Spanish Ministry of Science and Innovation (MICINN) project AYA2017-86389-P. J.I.G.H. acknowledges financial support from the Spanish MICINN under the 2013 Ramón y Cajal 
program RYC-2013-14875. M.T.M. thanks the Australian Research Council for Future Fellowship grant FT180100194 which supported this work. This publication makes use of the Data \& Analysis Center for Exoplanets (DACE), which is a facility based at the University of Geneva $(\mathrm{CH})$ dedicated to extrasolar planets data visualization, exchange and analysis. DACE is a platform of the Swiss National Centre of Competence in Research (NCCR) PlanetS, federating the Swiss expertise in Exoplanet research. The DACE platform is available at https://dace.unige.ch.

\section{References}

Aliverti, M., Pariani, G., Moschetti, M., \& Riva, M. 2016, in Integration and Alignment Through Mechanical Measurements: The Example of the ESPRESSO Front-end Units, SPIE Conf. Ser., 9908, 99087C

Álvarez, D., Lizon, J. L., Hughes, I., et al. 2018, in SPIE Conf. Ser., Proc. SPIE, 10702, 107026W

Alves, C., Silva, T., Martins, C., \& Leite, A. 2017, Phys. Lett. B, 770, 93

Anglada-Escudé, G., Amado, P. J., Barnes, J., et al. 2016, Nature, 536, 437

Arentsen, A., Starkenburg, E., Shetrone, M. D., et al. 2019, A\&A, 621, A108

Arns, J. A. 2016, in Performance Characteristics of Two Volume Phase Holographic Grisms Produced for the ESPRESSO Spectrograph, SPIE Conf. Ser., 9908, 990861

Avila, G., Cabral, A., Coelho, J. P., et al. 2016, in Alignment of the ESPRESSO Coudé train on the ESO VLT, SPIE Conf. Ser., 9912, 99124L

Bahcall, J. N., \& Schmidt, M. 1967, Phys. Rev. Lett., 19, 1294

Bainbridge, M. B., \& Webb, J. K. 2017, MNRAS, 468, 1639

Baldini, V., Calderone, G., Cirami, R., et al. 2016, in Integration of the Instrument Control Electronics for the ESPRESSO Spectrograph at ESO VLT, SPIE Conf. Ser., 9913, 99132H

Baranne, A., Queloz, D., Mayor, M., et al. 1996, A\&AS, 119, 373

Batalha, N. M., Rowe, J. F., Bryson, S. T., et al. 2013, ApJS, 204, 24

Bonifacio, P., Rahmani, H., Whitmore, J. B., et al. 2014, Astron. Nachr., 335, 83

Bonifacio, P., Molaro, P., Adibekyan, V., et al. 2020, A\&A, 633, A129

Borucki, W. J., Koch, D. G., Basri, G., et al. 2011, ApJ, 736, 19

Bouchy, F., Pepe, F., \& Queloz, D. 2001, A\&A, 374, 733

Buchhave, L. A., Dressing, C. D., Dumusque, X., et al. 2016, AJ, 152, 160

Cabral, A., Coelho, J., Abreu, M., et al. 2013, in Optical Design of a CoudéTrain for a Stable and Efficient Simultaneous Feeding of the ESPRESSO Spectrograph from the Four VLT Telescopes, SPIE Conf. Ser., 8785, 87850L

Cabral, A., Abreu, M., Coelho, J., et al. 2014, in ESPRESSO Coudé-Train Complexities of a Simultaneous Optical Feeding from the Four VLT Unit Telescopes, SPIE Conf. Ser., 9147, 91478Q

Cabral, A., Abreu, M., Coelho, J., et al. 2019, in IV International Conference on Applications of Optics and Photonics AOP, Proc. SPIE, 11207, 1120701

Caffau, E., Bonifacio, P., François, P., et al. 2011, Nature, 477, 67

Calderone, G., Baldini, V., Cirami, R., et al. 2016, in The Technical CCDs in ESPRESSO: Usage, Performances, and Network Requirements, SPIE Conf. Ser., 9913, 99132K

Calderone, G., Baldini, V., Cirami, R., et al. 2018, in SPIE Conf. Ser., Proc. SPIE, 10707, 107072G

Carrier, F., \& Bourban, G. 2003, A\&A, 406, L23

Carroll, S. M. 1998, Phys. Rev. Lett., 81, 3067

Casasayas-Barris, N., Pallé, E., Yan, F., et al. 2019, A\&A, 628, A9

Cersullo, F., Coffinet, A., Chazelas, B., Lovis, C., \& Pepe, F. 2019, A\&A, 624, A122

Charbonneau, D., Brown, T. M., Noyes, R. W., \& Gilliland, R. L. 2002, ApJ, 568,377

Chazelas, B., Pepe, F., Wildi, F., et al. 2010, in New Scramblers for Precision Radial Velocity: Square and Octagonal Fibers, SPIE Conf. Ser., 7739, 773947

Chazelas, B., Pepe, F., \& Wildi, F. 2012, in Optical Fibers for Precise Radia Velocities: An Update, SPIE Conf. Ser., 8450, 845013

Cooke, R. J., Pettini, M., \& Steidel, C. C. 2018, ApJ, 855, 102

Cosentino, R., Lovis, C., Pepe, F., et al. 2012, in Harps-N: The New Planet Hunter at TNG, SPIE Conf. Ser., 8446, 84461V

Cristiani, S., Avila, G., Bonifacio, P., et al. 2007, Nuovo Cimento B Serie, 122, 1165

Cunha, D., Figueira, P., Santos, N. C., Lovis, C., \& Boué, G. 2013, A\&A, 550, A75

Cunha, D., Santos, N. C., Figueira, P., et al. 2014, A\&A, 568, A35

Cupani, G., D’Odorico, V., Cristiani, S., et al. 2016, in SPIE Conf. Ser., Proc. SPIE, 9913, 99131T

Cupani, G., Calderone, G., Cristiani, S., et al. 2018, in SPIE Conf. Ser., Proc. SPIE, 10707, 1070723

Cupani, G., D’Odorico, V., Cristiani, S., et al. 2019, in Astronomical Data Analysis Software and Systems XXVI, eds. M. Molinaro, K. Shortridge, \& F Pasian, ASP Conf. Ser., 521, 362

Díaz, R. F., Ségransan, D., Udry, S., et al. 2016, A\&A, 585, A134
Di Marcantonio, P., Sosnowska, D., Cupani, G., et al. 2018, in SPIE Conf. Ser., Proc. SPIE, 10704, 107040F

D’Odorico, V., Cristiani, S., D’Odorico, S., et al. 1998, A\&A, 339, 678

D'Odorico, V., Petitjean, P., \& Cristiani, S. 2002, A\&A, 390, 13

Dumusque, X. 2012, PhD Thesis, Observatory of Geneva, Switzerland

Ehrenreich, D., Lovis, C., Allart, R., et al. 2020, Nature, 580, 597

Evans, T., Murphy, M. T., Whitmore, J. B., et al. 2014, MNRAS, 445, 128

Findlay, J. R., Prochaska, J. X., Hennawi, J. F., et al. 2018, ApJS, 236, 44

Frank, C., Kerber, F., Avila, G., et al. 2018, in SPIE Conf. Ser., Proc. SPIE, 10702, 107026P

Fulton, B. J., Petigura, E. A., Howard, A. W., et al. 2017, AJ, 154, 109

Gandolfi, D., Barragán, O., Livingston, J. H., et al. 2018, A\&A, 619, L10

Genoni, M., Riva, M., Pariani, G., Aliverti, M., \& Moschetti, M. 2016, in Spectrograph Sensitivity Analysis: An Efficient Tool for Different Design Phases, SPIE Conf. Ser., 9908, $99086 Z$

González Hernández, J. I., Pepe, F., Molaro, P., \& Santos, N. C. 2018, ESPRESSO on VLT: An Instrument for Exoplanet Research, 157

Gracia Temich, F., Rasilla, J. L., \& Salata, S. 2018a, in SPIE Conf. Ser., Proc. SPIE, 10702, 1070273

Gracia Temich, F., Rasilla, J. L., Salata, S., et al. 2018b, in SPIE Conf. Ser., Proc. SPIE, 10706, 1070628

Greif, T. H., Springel, V., White, S. D. M., et al. 2011, ApJ, 737, 75

Heger, A., \& Woosley, S. E. 2010, ApJ, 724, 341

Hoeijmakers, H. J., Ehrenreich, D., Kitzmann, D., et al. 2019, A\&A, 627, A165

Hojjatpanah, S., Figueira, P., Santos, N. C., et al. 2019, A\&A, 629, A80

Huang, C. X., Burt, J., Vanderburg, A., et al. 2018, ApJ, 868, L39

Huke, P., Schäfer, S., Reiners, A., et al. 2018, in SPIE Conf. Ser., Proc. SPIE, 10702, 107029L

King, J. A., Webb, J. K., Murphy, M. T., et al. 2012, MNRAS, 422, 3370

Kjeldsen, H., \& Bedding, T. R. 2011, A\&A, 529, L8

Kjeldsen, H., Bedding, T. R., Butler, R. P., et al. 2005, ApJ, 635, 1281

Kotus, S. M., Murphy, M. T., \& Carswell, R. F. 2017, MNRAS, 464, 3679

Landoni, M., Riva, M., Pepe, F., et al. 2014, in ESPRESSO Front End Exposure Meter: A Chromatic Approach to Radial Velocity Correction, SPIE Conf. Ser., 9147, 91478K

Landoni, M., Riva, M., Pepe, F., et al. 2016, in ESPRESSO Front End Guiding Algorithms: From Design Phase to Implementation and Validation Toward the Commissioning, SPIE Conf. Ser., 9913, 99133Q

Leite, A., Martins, C., Molaro, P., Corre, D., \& Cristiani, S. 2016, Phys. Rev. D, 94, 123512

Levshakov, S. A., Reimers, D., Henkel, C., et al. 2013, A\&A, 559, A91

Li, Z., Wolf, C., Bian, F., et al. 2018, PASA, submitted [arXiv:1805.03429]

Liske, J., Grazian, A., Vanzella, E., et al. 2008, MNRAS, 386, 1192

Lizon, J. L., Iwert, O., Deiries, S., et al. 2016, in An Ultra-Stable Cryostat for the Detectors of ESPRESSO, SPIE Conf. Ser., 9908, 990866

Lizon, J. L., Dekker, H., Manescau, A., et al. 2018, in SPIE Conf. Ser., Proc. SPIE, 10701, 107012P

Lo Curto, G., Pepe, F., Avila, G., et al. 2015, The Messenger, 162, 9

Loeb, A. 1998, ApJ, 499, L111

Lovis, C., Ségransan, D., Mayor, M., et al. 2011, A\&A, 528, A112

Martins, C. 2017, Rep. Progr. Phys., 80, 126902

Martins, C., \& Vacher, L. 2019, Phys. Rev. D, 100, 123514

Martins, J. H. C., Santos, N. C., Figueira, P., et al. 2015a, A\&A, 576, A134

Martins, C., Pinho, A., Alves, R., et al. 2015b, JCAP, 08, 047

Mayor, M., \& Queloz, D. 1995, Nature, 378, 355

Mayor, M., Pepe, F., Queloz, D., et al. 2003, The Messenger, 114, 20

Mégevand, D., Zerbi, F. M., Di Marcantonio, P., et al. 2014, in ESPRESSO: The Radial Velocity Machine for the VLT, SPIE Conf. Ser., 9147, 91471H

Molaro, P. 2009, Astrophys. Space Sci. Proc., 9, 389

Molaro, P., Murphy, M. T., \& Levshakov, S. A. 2006, in The Scientific Requirements for Extremely Large Telescopes, eds. P. Whitelock, M. Dennefeld, \& B. Leibundgut, IAU Symp., 232, 198

Molaro, P., Centurión, M., Whitmore, J. B., et al. 2013, A\&A, 555, A68

Murphy, M. T., \& Cooksey, K. L. 2017, MNRAS, 471, 4930

Murphy, M. T., Flambaum, V. V., Webb, J. K., et al. 2004, in Constraining Variations in the Fine-Structure Constant, Quark Masses and the Strong Interaction, eds. S. G. Karshenboim, \& E. Peik, 648, 131

Murphy, M. T., Malec, A. L., \& Prochaska, J. X. 2016, MNRAS, 461, 2461

Nortmann, L., Pallé, E., Salz, M., et al. 2018, Science, 362, 1388

Oggioni, L., Pariani, G., Moschetti, M., et al. 2016, in MMP, The Multi Mini Prism Device for ESPRESSO APSU: Prototyping and Integration, SPIE Conf. Ser., 9908, 990872

Pariani, G., Aliverti, M., Moschetti, M., et al. 2016, in Integration, Alignment, and Verification of the ESPRESSO Front-End, SPIE Conf. Ser., 9908, 99087B

Pariani, G., Aliverti, M., Genoni, M., et al. 2018, in SPIE Conf. Ser., Proc. SPIE, 10706, 107064H

Pasquini, L., \& Hubin, N. 2018, in SPIE Conf. Ser., Proc. SPIE, 10702, 1070204 
Pasquini, L., Cristiani, S., Dekker, H., et al. 2005, The Messenger, 122, 10

Pasquini, L., Manescau, A., Avila, G., et al. 2009, Astrophys. Space Sci. Proc., 9, 395

Pepe, F., Mayor, M., Rupprecht, G., et al. 2002, The Messenger, 110, 9

Pepe, F., Lovis, C., Ségransan, D., et al. 2011, A\&A, 534, A58

Pepe, F., Molaro, P., Cristiani, S., et al. 2014, Astron. Nachr., 335, 8

Perryman, M., Hainaut, O., Dravins, D., et al. 2005, ESA-ESO Working Group on "Extra-solar Planets" (ESA)

Queloz, D., Mayor, M., Weber, L., et al. 2000, A\&A, 354, 99

Rahmani, H., Wendt, M., Srianand, R., et al. 2013, MNRAS, 435, 861

Rauch, M., Becker, G. D., Viel, M., et al. 2005, ApJ, 632, 58

Redfield, S., Endl, M., Cochran, W. D., \& Koesterke, L. 2008, ApJ, 673, L87

Ricker, G. R., Winn, J. N., Vanderspek, R., et al. 2015, J. Astron. Telesc. Instrum. Syst., 1, 014003

Riemer-Sørensen, S., Webb, J. K., Crighton, N., et al. 2015, MNRAS, 447, 2925

Riemer-Sørensen, S., Kotuš, S., Webb, J. K., et al. 2017, MNRAS, 468, 3239

Riva, M., Aliverti, M., Moschetti, M., et al. 2014a, in ESPRESSO Front End: Modular Opto-Mechanical Integration for Astronomical Instrumentation, SPIE Conf. Ser., 9147, 91477G

Riva, M., Conconi, P., Moschetti, M., et al. 2014b, in APSU at ESPRESSO: Final Design Towards the Integration, SPIE Conf. Ser., 9147, 91477D

Rosenband, T., Hume, D. B., Schmidt, P. O., et al. 2008, Science, 319, 1808

Roy, A., Halverson, S., Mahadevan, S., et al. 2020, AJ, 159, 161

Santana Tschudi, S., Fragoso, A., Amate, M., et al. 2014, in Design of the OptoMechanical Mounts of the ESPRESSO Spectograph, SPIE Conf. Ser., 9151 915153

Santos, N. C., Adibekyan, V., Dorn, C., et al. 2017, A\&A, 608, A94

Snellen, I. A. G., Albrecht, S., de Mooij, E. J. W., \& Le Poole, R. S. 2008, A\&A, 487,357

Sotzen, K. S., Stevenson, K. B., Sing, D. K., et al. 2020, AJ, 159, 5

Sousa, S. G., Santos, N. C., Israelian, G., et al. 2007, A\&A, 469, 783

Sousa, S. G., Santos, N. C., Adibekyan, V., Delgado-Mena, E., \& Israelian, G. 2015, A\&A, 577, A67

Suárez Mascareño, A., Faria, J. P., Figueira, P., et al. 2020, A\&A, 639, A77

Tenegi, F., Santana, S., Gómez, J., et al. 2016, in ESPRESSO Optical Bench: From Mind to Reality, SPIE Conf. Ser., 9912, 99123K

Thompson, R. I. 1975, Astrophys. Lett., 16, 3

Udry, S., Dumusque, X., Lovis, C., et al. 2019, A\&A, 622, A37

Uzan, J.-P. 2011, Liv. Rev. Relativ., 14, 2

Van Eylen, V., Agentoft, C., Lundkvist, M. S., et al. 2018, MNRAS, 479, 4786

Vanzella, E., Meneghetti, M., Pastorello, A., et al. 2020, MNRAS, 499, L67

Webb, J. K., Flambaum, V. V., Churchill, C. W., Drinkwater, M. J., \& Barrow, J. D. 1999, Phys. Rev. Lett., 82, 884

Webb, J. K., King, J. A., Murphy, M. T., et al. 2011, Phys. Rev. Lett., 107, 191101

Wyttenbach, A., Lovis, C., Ehrenreich, D., et al. 2017, A\&A, 602, A36
1 Département d'Astronomie, Université de Genève, Ch. des Maillettes 51, 1290 Versoix, Switzerland e-mail: francesco.pepe@unige.ch

2 INAF - Osservatorio Astronomico di Trieste, Via Tiepolo 11, 34143 Trieste, Italy

3 Instituto de Astrofisica de Canarias, Via Lactea, 38200 La Laguna, Tenerife, Spain

${ }^{4}$ Instituto de Astrofísica e Ciências do Espaço, Universidade do Porto, CAUP, Rua das Estrelas, 4150-762 Porto, Portugal

5 ESO, European Southern Observatory, Karl-Schwarzschild-Straße 2, 85748 Garching, Germany

${ }^{6}$ Instituto de Astrofísica e Ciências do Espaço, Universidade de Lisboa, Edifício C8, 1749-016 Lisboa, Portugal

7 INAF - Osservatorio Astronomico di Brera, Via Bianchi 46, 23807 Merate, Italy

${ }^{8}$ Centro de Astrobiología (CSIC-INTA), Carretera de Ajalvir km 4, 28850 Torrejón de Ardoz, Madrid, Spain

9 Physics Institute of University of Bern, Gesellschaftsstrasse 6, 3012 Bern, Switzerland

10 INAF - Osservatorio Astrofisico di Torino, Via Osservatorio 20, 10025 Pino Torinese, Italy

11 Departamento de Física da Faculdade de Ciências da Universidade de Lisboa, Edifício C8, 1749-016 Lisboa, Portugal

12 Departamento de Física e Astronomia, Faculdade de Ciências, Universidade do Porto, Rua do Campo Alegre, 4169-007 Porto, Portugal

13 INAF - Osservatorio Astronomico di Palermo, Piazza del Parlamento 1, 90134 Palermo, Italy

14 NRCC-HIA, 5071 West Saanich Road Building VIC-10, Victoria, British Columbia V9E 2E, Canada

15 ESO, European Southern Observatory, Alonso de Cordova 3107, Vitacura, Santiago, Chile

16 Institute for Fundamental Physics of the Universe, IFPU, Via Beirut 2, 34151 Grignano, Trieste, Italy

17 Centro de Astrofísica da Universidade do Porto, Rua das Estrelas, 4150-762 Porto, Portugal

18 Universidad de La Laguna, Departamento de Astrofísica, 38206 La Laguna, Tenerife, Spain

19 Consejo Superior de Investigaciones Científicas, 28006 Madrid, Spain

20 Centre for Astrophysics and Supercomputing, Swinburne University of Technology, Hawthorn, Victoria 3122, Australia 